\title{
ty- \\ Plantas que mantienen al ganado: conocimiento CAMPESINO ASOCIADO A ESPECIES FORRAJERAS EN LA Sierra de Ancasti (Catamarca, Argentina)
}

\author{
Plants to Raise livestock: Peasant knowledge associated to fodder \\ species in Sierra de Ancasti (Catamarca, Argentina)
}

\author{
N. David Jiménez-Escobar ${ }^{1 *}$ (i) y Gustavo J. Martínez ${ }^{1}[0$
}

1. IDACOR-CONICET, Museo de Antropología, FFyH, Universidad Nacional de Córdoba. Hipólito Yrigoyen 174, Córdoba, Argentina.

*ndjimeneze@gmail.com

Citar este artículo JIMÉNEZ-ESCOBAR, N. D. \& G. J. MARTÍNEZ. 2019. Plantas que mantienen al ganado: conocimiento campesino asociado a especies forrajeras en la Sierra de Ancasti (Catamarca, Argentina). Bol. Soc. Argent. Bot. 54: 617-635.

DOI: http://dx.doi. org/10.31055/1851.2372.v54. n4.24707

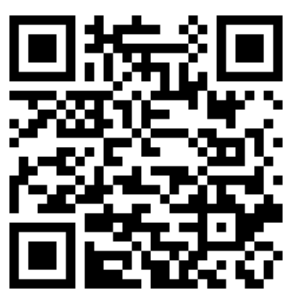

Recibido: 13 Junio 2019 Aceptado: 1 Octubre 2019 Publicado: 15 Diciembre 2019 Editora: Norma Hilgert (iD

ISSN versión impresa 0373-580X ISSN versión on-line 1851-2372

\section{SUMMARY}

Background and aims: Peasant communities associated with small-scale livestock often have deep knowledge of the environment, ecosystems and plant species in which their animals are fed. In this study, knowledge associated with forage plants in the Sierra de Ancasti, Argentina (Dry Chaco) was inquired. Accordingly, we determined what types of livestock is raised in the area, what plants are consumed by domestic animals and, in local terms, what is considered a forage.

M\&M: By using the snowball technique, 20 households, related to livestock areas, participated in the open and semi-structured interviews. Study participants were specifically asked about forage species. Field work was complemented with participant observation and guided walks with peasant shepherd families related.

Results: Local production is mainly related to goats, sheep and cattle. Regarding forage plants, 154 species were found (corresponding to 129 genera, 51 families). The high percentage of species of native origin is significant (78\%). According to the Frequency Index, the most widely cited kinds of forage are: Cereus forbesii, Medicago sativa, Vachellia aroma, V. caven y Zea mays.

Conclusions: The variety of species consumed by the different types of livestock, the prevalence of native fodder species and the diversity of habits and parts used highlights the region's rich biological diversity. Likewise, the forage term was defined by Ancasti's inhabitants as associated with the concept of "raise" the animal (feeding, fattening, caring or increasing production).

\section{KEY WORDS}

Dry Chaco, ethnobotany, fodder plants, livestock, rural communities.

\section{REsumen}

Introducción y objetivos: Las comunidades campesinas asociadas al pastoreo y a la ganadería -a pequeña escala- suelen tener un profundo conocimiento de los ambientes, de los ecosistemas y de las plantas en las que se crían sus animales. En este estudio se indagó el conocimiento asociado a las plantas forrajeras en la Sierra de Ancasti, Argentina (Chaco seco). Para ello se estableció qué tipo de ganado se cría en la zona, qué plantas son consumidas por los animales domésticos y en términos locales, qué se considera un forraje.

M\&M: Por medio de la técnica bola de nieve, 20 unidades domésticas participaron de las entrevistas abiertas y semiestructuradas, consultándoles específicamente por las especies forrajeras. El trabajo de campo se complementó con observación participante y caminatas guiadas junto a familias campesinas asociadas al pastoreo.

Resultados: La producción local se relaciona principalmente al ganado caprino, ovino y vacuno. En relación a las plantas forrajeras, se encontraron 154 especies (correspondientes a 129 géneros, 51 familias). Se destaca el alto porcentaje de especies de origen nativo (78\%). Según el Índice de frecuencia de mención, los forrajes más citados son: Cereus forbesii, Medicago sativa, Vachellia aroma, V. caven y Zea mays.

Conclusiones: La riqueza de especies consumidas por los distintos tipos de ganado, el dominio de las especies nativas en la alimentación de los animales y la diversidad de tipos de hábito y partes de uso, son una muestra de la amplia diversidad biológica de la región. Asimismo, se definió el término de forraje, que para los pobladores de Ancasti está asociado al concepto de "mantener" al animal (alimentar, engordar, cuidar, aumentar la producción).

\section{Palabras clave}

Chaco Seco, etnobotánica, forrajes, ganadería, poblaciones rurales. 


\section{INTRODUCCIÓN}

El pastoreo y la ganadería -a pequeña escalase puede definir como la cría, el cuidado y el manejo del ganado en espacios abiertos o al aire libre (Galaty \& Johnson, 1990). Alrededor del mundo, este tipo de ganadería -a pequeña escalaconstituye para sociedades y pueblos pastoriles, la principal fuente de producción, donde la fuerza laboral gira en torno al cuidado animal y genera estabilidad económica a los pobladores (Galaty \& Johnson, 1990; De Rancourt et al., 2006; Scarpa, 2012; Rivera, 2014; Nunes et al., 2015). De esta manera, el ganado no solo forma parte del capital financiero de las unidades domésticas, también constituye una fuente de ahorro y de recursos que se hacen disponibles en situaciones de emergencia o necesidad (Rivera, 2014).

Para las comunidades indígenas y campesinas, la cría de animales involucra una serie de dinámicas y prácticas, que van más allá de los de los ámbitos productivos, donde el ganado también hace parte de numerosas redes simbólicas y sociales, que permean el día a día de las comunidades, otorgándoles características propias a las unidades familiares y ocupando lugares de privilegio en su cultura (EvanPritchard, 1940; Schwabe \& Kuojok, 1981; Galaty \& Johnson, 1990; Quiroga Mendiola, 2011; Scarpa, 2012).

En efecto, la cría de animales es una práctica antigua que representa una importante fuente de subsistencia para los hogares con bajos ingresos en todo el mundo (UiCN, 2008; FAO, 2011; Galeano et al., 2013; Nunes et al., 2015). El ganado también permite a los pobladores, la diversificación de sus propios recursos, amainando los riesgos frente a los cambios sociales, económicos y climáticos (Rivera, 2014). De esta forma, en los ambientes áridos y semiáridos, donde la producción agrícola es escasa, el pastoreo se ha constituido como una práctica frecuente y propia de las comunidades humanas que habitan estos ecosistemas (FAO, 2011). El Chaco Seco no escapa a esta realidad, siendo frecuente entre los pobladores la crianza principalmente de ganado caprino, pero también de ovino y vacuno (Roig, 2003; Bazán, 2006; Cáceres, 2006; Scarpa, 2007, 2012; Ramisch et al., 2009; Cavanna et al., 2010; Riat, 2012; Jiménez-Escobar, 2015, 2019).

Por definición la palabra forraje, es referida a aquellas partes vegetales consumidas por el ganado
(Ledesma et al., 2007). En la Argentina, uno de los primeros autores que resaltó la importancia de las investigaciones sobre plantas forrajeras fue Burkart (1943), quien afirma que las comunidades ganaderas, presentan un "caudal de conocimientos", donde se distinguen los "mejores pastos", sus dominios y su valor forrajero. Luego se han publicado otros trabajos que abordan el estudio de las prácticas ganaderas y la dieta vacuna en el Chaco Argentino (Morello \& Saravia, 1959a, b; Ragonese, 1967; Morello et al., 1973; Ragonese \& Milano, 1984; Braun Wilke, 1995; Roig, 2003). Asimismo, el estudio del uso de pastizales naturales y sabanas como ecosistemas para el desarrollo de la ganadería ha motivado el interés de otras investigaciones (Marino, 2008, 2013). Por otra parte, resultan pioneros los estudios de sustentabilidad ecológica y socio-cultural para ecosistemas pastoriles en ambientes áridos de alta montaña, realizados por Quiroga-Mendiola (2011) en la provincia de Jujuy.

Para la etnobiología, se destacan los aportes de Scarpa (2007) quien, para el oeste de Formosa, cita 196 taxas vegetales y construye un Índice de Valor Forrajero (Ivf) para calcular la calidad y la disponibilidad de 189 plantas forrajeras silvestres. Por otra parte, para la Provincia de Santiago del Estero el trabajo de Riat (2012), presenta 35 especies vegetales reconocidas como forrajes. Mientras para la provincia de Catamarca, Cavanna et al. (2010) realizaron una primera descripción de las plantas forrajeras con comunidades campesinas de las Salinas Grandes y Jiménez-Escobar (2015) destacó el uso y manejo de 14 especies de la familia Bromeliaceae, consumidas por el ganado en áreas serranas.

Las comunidades campesinas asociadas a la cría de animales suelen tener un profundo conocimiento de los ambientes, del ecosistema y de las plantas. Estos saberes populares se revisten de gran valor social, económico y cultural, principalmente en lugares donde la alimentación de los animales de cría depende de las pasturas naturales y del bosque nativo. Por lo tanto, para una planificación ganadera sostenible y una valoración de los recursos vegetales asociados a estos espacios, es necesario establecer la riqueza de plantas vinculadas a las prácticas de pastoreo. De igual manera, se debe evaluar el uso y el aprovechamiento de algunas especies, las formas de manejo y el tipo de ganado. Estos lineamientos deben considerarse como herramientas importantes 


\section{N. D. Jiménez-Escobar y G. J. Martínez - Plantas forrajeras de la Sierra de Ancasti, Catamarca}

a la hora de proponer estrategias de conservación, dado el impacto que generan sobre las poblaciones vegetales y en general sobre la conformación del paisaje. En este contexto, las indagaciones que se pretenden contestar, están relacionadas directamente a los pequeños y medianos productores ganaderos, con quienes se abordaron los siguientes cuestionamientos: ¿Qué es un forraje en términos locales? ¿Qué tipos de ganado se crían en Ancasti? ¿Cuáles plantas son consumidas por los animales?

\section{Materiales y Métodos}

\section{Área de estudio}

El departamento de Ancasti está situado entre $\operatorname{los} 28^{\circ} 23^{\prime} 29,3^{\prime \prime} \mathrm{S}$ y los $65^{\circ} 20^{\prime} 65,4^{\prime \prime} \mathrm{O}$. Posee una superficie de $2.412 \mathrm{Km}^{2}$ lo que representa el 2,35\% de la dimensión geográfica de la provincia de Catamarca (Fig. 1). La Sierra de Ancasti está dentro de la provincia geográfica chaqueña (distrito chacoserrano). Se caracteriza por presentar un clima cálidohúmedo, con una temperatura media anual de $19^{\circ} \mathrm{C}$ y precipitaciones que oscilan entre los 500 y $1200 \mathrm{~mm}$ anuales, concentradas en la época de verano (Bazán, 2006; Palmeri et al., 2008).

Con un relieve montañoso, la región presenta praderas de altura con pasturas naturales, quebradas, pequeños valles intermontanos y faldeos con formaciones boscosa. La estructura vegetal de la zona se caracteriza por combinar elementos arbóreos típicos del Chaco Seco, como Celtis spp., Geoffroea decorticans (Gillies ex Hook. \& Arn) Burkart., Jodina rhombifolia (Hook. \& Arn.) Reissek y Schinopsis lorentzii (Griseb.) Engl, con elementos propios del Chaco Serrano, como Lithraea molleoides (Vell.) Engl., Parapiptadenia excelsa (Griseb.) Burkart, Parasenegalia visco (Lorentz ex Griseb.) Seigler \& Ebinger, Ruprechtia apetala Wedd. y Zanthoxylum coco Gillies ex Hook. f. \& Arn., y especies asociadas a la eco-región Yungas (en Ancasti, selva de transición o cebilar) como Anadenanthera colubrina (Vell.) Brenan, Erythroxylum argentinum O.E. Schulz y Xylosma pubescens Griseb.

Las praderas de altura o pastizales de neblina son uno de los paisajes más representativos de la Sierra (1000 -1200 msnm). Presentan una marcada estacionalidad, donde se destaca un amplio dominio de pastizales nativos (Cyperus corymbosus Rottb., Elionurus muticus (Spreng.) Kuntze, Festuca hieronymi Hack., Jarava ichu Ruiz \& Pav., Poa calchaquiensis Hack., Setaria macrostachya Kunth, solo por destacar algunos), con elementos de porte herbáceo a arbustivo (Baccharis flabellata Hook. \& Arn., Berberis ruscifolia Lam., Croton lachnostachyus Baill. y Ephedra tweediana Fisch. \& C.A. Mey.) y la ausencia de árboles. En relación con la ecología vegetal y la vegetación de los ambientes del Este de Catamarca, se pueden reseñar las investigaciones referentes a la fitogeografía (Morláns, 1995), la flora (Perea et al., 2007; Quiroga \& Reinoso-Franchino, 2010; Perea, 2011) y las gramíneas forrajeras del Chaco seco (Quiroga \& Correa 2011). Asimismo, recientes contribuciones incluyen percepciones y usos de plantas por parte de la comunidad rural de Ancasti (Jiménez-Escobar, 2015, 2019; Martínez \& JiménezEscobar, 2017; Jiménez- Escobar \& Martínez, 2019)

\section{Pobladores actuales de la Sierra}

Según los datos más recientes datos del Instituto Nacional de Estadísticas y Censos (INDEC, 2010), el departamento está conformado por una población de 2900 habitantes, que conforman un total de 786 hogares o unidades familiares. La mayoría de estos pobladores viven en casas o departamentos (91\%), siendo muy bajo el porcentaje de familias que habita en otro tipo de vivienda como ranchos (7\%) o casillas (1\%). Según Ramisch et al. (2009), en el año 2002 en Ancasti existían cerca de 494 explotaciones agropecuarias, de las cuales 450 (91\%) eran conformadas por pequeños productores familiares.

En cuanto a la jurisdicción política de Ancasti, el departamento se divide en 10 distritos, donde están asentadas poblaciones con escasa concentración demográfica, siendo el único municipio la Villa de Ancasti (305 habitantes), mientras la segunda localidad en importancia poblacional es Anquincila (200 habitantes). El resto de la población esta diseminada en caseríos y parajes que conforman la típica fisonomía rural de la zona (INDEC, 2010).

En la actualidad, los pobladores rurales de la región que se autodefinen como criollos-serranos y mantienen una clara vocación campesina (JiménezEscobar, 2019). La economía de subsistencia sigue siendo y está asociada principalmente a sistemas a campo abierto de producción ganadera a pequeña escala. Según Ramisch et al., (2009) aunque en la región hay una importante producción de ganado vacuno, Ancasti se caracteriza por tener una producción caprina y ovina predominante. En la 
Sierra, la agricultura cuando es a escala familiar, está relegada generalmente a pequeñas chacras, en donde se cultiva principalmente "maíz" (Zea mays L.) y "zapallo" (Cucurbita maxima Duch.).

\section{Fase de campo}

Los datos etnobotánicos específicos para esta temática fueron obtenidos a través de 4 campañas de campo, realizadas entre junio de 2016 y marzo de 2018. Asimismo, se sistematizó la información general obtenida por el equipo de etnobiología CONICET-IDACOR, Museo de Antropología, FFyH-UNC, que desde el año 2001, viene realizando caminatas etnobotánicas de reconocimiento de especies a campo y entrevistas abiertas y en profundidad (Albuquerque et al., 2014). El enfoque en temáticas forrajeras se llevó a cabo con pequeños productores ganaderos de la zona. Previo a las entrevistas con los pobladores, se informó acerca del proyecto de investigación y sus objetivos a

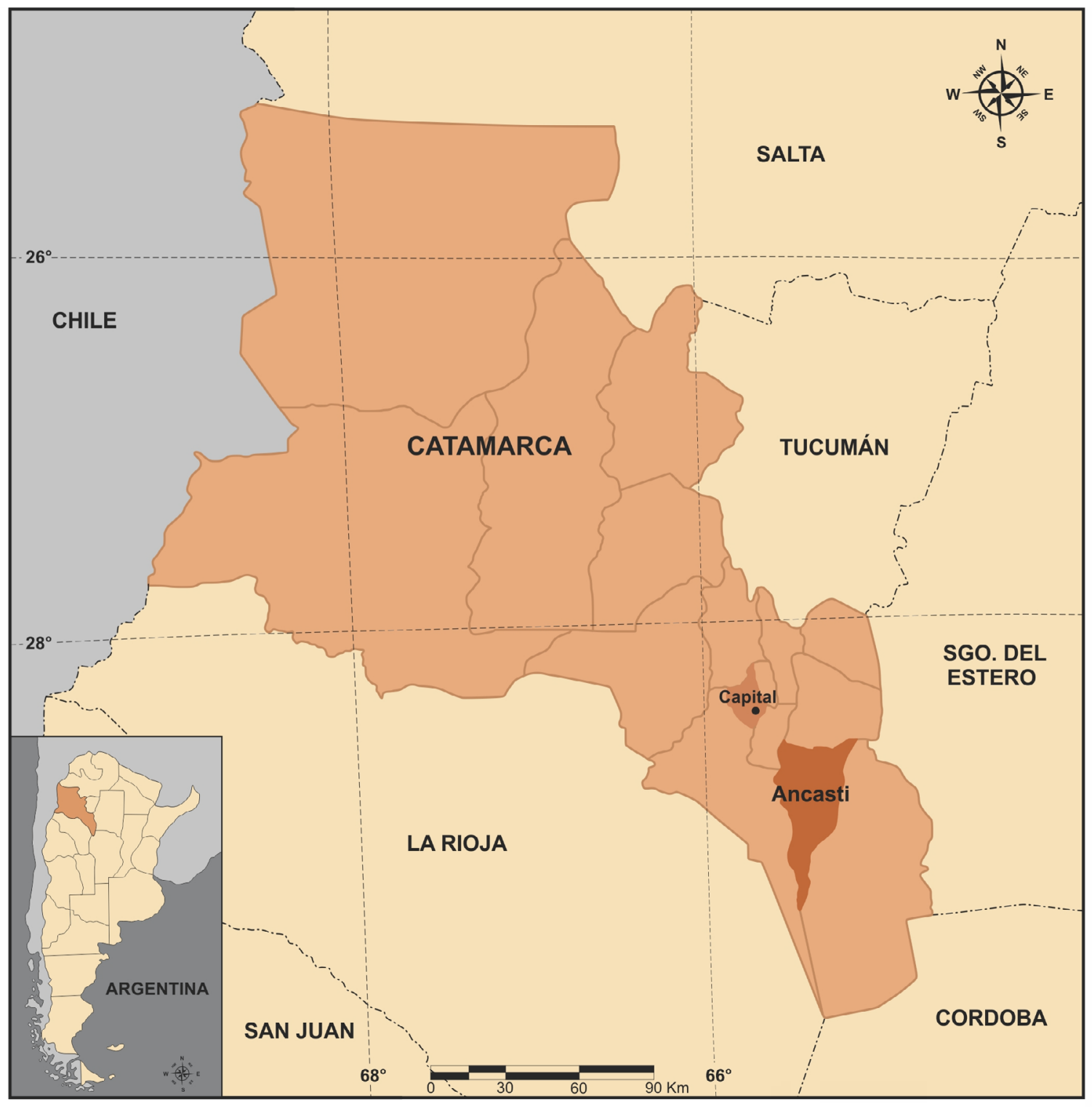

Fig. 1. Ubicación del área de estudio, Departamento de Ancasti, Provincia de Catamarca, Argentina. 


\section{N. D. Jiménez-Escobar y G. J. Martínez - Plantas forrajeras de la Sierra de Ancasti, Catamarca}

los representantes municipales de Ancasti y se estableció un convenio de investigación con la Secretaría de Estado del Ambiente y Desarrollo Sustentable, Gobernación de la Provincia de Catamarca (Expediente $\mathrm{N}^{\circ}$ 28950/15). De igual forma, en cada una de las unidades familiares visitadas se socializó el proyecto, así como sus alcances y por medio de consentimiento verbal se solicitó a los pobladores su colaboración, en concordancia con el Código de Ética de la Sociedad Internacional de Etnobiología (ISE 2006).

En total, 20 unidades domésticas (U.D.) participaron de las entrevistas abiertas y semiestructuradas donde seconsultó específicamente por las especies forrajeras involucradas en el manejo ganadero (Karremans, 1994; Albuquerque et al., 2014). Asimismo, se enfatizó en temas relacionados a la alimentación animal (forrajes: nativos, usados, adquiridos o comprados), especies consideradas de importancia, criterios de preferencia en el animal, partes consumidas, tipos de ganado, entre otras.

Paralelamente y de forma complementaria, con la técnica de observación participante (Guber, 2004, 2011), se realizaron registros etnográficos en tres unidades familiares que presentaron diferentes tipos de ganado y cuya actividad económica central es dependiente de la cría de animales. Acompañados por los pastores de estas unidades familiares se realizaron caminatas guiadas, recorridas al campo y recolección de ejemplares vegetales (Albuquerque et al., 2014). Con estas familias también se compartieron varias actividades socio-culturales, vinculadas específicamente al manejo ganadero, tales como: el esquile de las ovejas, la marcada del ganado vacuno (la hierra o la yerra), las pariciones de animales de cría, la recolección de forrajes en el invierno, la cosecha del maíz, la alimentación con forrajes comprados, las cabalgatas como forma de locomoción, la faena de corderos, entre otras.

\section{Procesamiento de material vegetal y análisis}

Las plantas fueron recolectadas principalmente en estado reproductivo, para su posterior identificación. La colección vegetal fue procesada según los estándares establecidos (Mori et al., 2011). Los ejemplares fueron determinados por los autores y posteriormente depositados en el Herbario del Museo Botánico (CORD), del Instituto Multidisciplinario de Biología Vegetal (IMBIV), Universidad Nacional de Córdoba,
Argentina, bajo la serie de numeración de los autores (GJM: Martínez y NDJ: Jiménez-Escobar, Tabla 1). La lista de especies sigue la propuesta taxonómica y nomenclatura establecida en el Catálogo de las Plantas Vasculares del Cono Sur, así como la información relacionada con el hábito y con el origen biogeográfico (Zuloaga et al., 2008, actualización [en línea]: www.darwin.edu.ar). Se realizó un análisis cualitativo de las narrativas y relatos acerca de las temáticas culturales, mientras que la información específicamente etnobotánica se sistematizó en una base de datos Excel, recurriendo para su análisis a la función tablas dinámicas.

Para establecer el origen biogeográfico de las especies, se recurrió al uso de dos categorías (nativa y exótica). Las especies nativas, fueron atribuidas en relación al área geográfica correspondiente a la provincia de Catamarca, siguiendo la propuesta de estatus de las especies vegetales publicado por el Catálogo de las Plantas Vasculares del Cono Sur (Zuloaga et al., 2008). La frecuencia de mención (Fr) corresponde al número de pobladores que citaron la especie dividida por el total de los entrevistados (20). Con la finalidad de poder realizar comparaciones, se siguen las consideraciones propuestas por Scarpa (2007) para el estudio de forrajes en Argentina.

\section{Resultados y Discusión}

\section{Forrajes de la Sierra de Ancasti}

En la Sierra de Ancasti los pobladores suelen diferenciar dos grandes categorías: las plantas forrajeras y las plantas que come el animal, aunque entre sí, estas dos categorías locales no son excluyentes y van a depender del tipo ganado que tiene el poblador y de la vegetación circundante. En general, un forraje es una o varias plantas que come el ganado, pero que además "mantiene" al animal, presentando alguna característica particular a la que se le atribuye algún beneficio y por la cual se destaca entre otras especies vegetales. Es así como, se dice en la Sierra: "Los forrajes no son todos, son aquellos que mantienen al animal". De esta manera, las forrajeras son plantas -o alguna de sus partes- que presentan una o varias cualidades, que se relacionan directamente con su cuidado: que no dejan morir de hambre al ganado en el invierno, que lo engordan en el verano, que le generan un aumento en la producción de leche, que lo comen 
las crías en sus primeros días de vida, que hacen "hueviar" a las aves de corral (aumento en la producción de huevos), entre otras.

\section{Tipos de ganado en la Sierra de Ancasti}

Los pobladores se caracterizan por criar principalmente tres tipos de ganado; el caprino (Capra aegagrus), el ovino (Ovis orientalis) y el vacuno (Bos taurus). Según Ramisch et al. (2009) aunque en la región hay una importante producción de ganado vacuno, Ancasti presenta una producción caprina y ovina predominante. Los animales pueden ser criados de manera combinada, aunque separados (vacas por un lado, cabras y ovejas por otro). En términos de Bugallo (2014), esta situación se relaciona con los requerimientos nutricionales distintos para cada especie y las capacidades que presentan en cuanto a la adaptación a pasturas y forrajes, por lo que demandan cuidados distintos.

La ganadería en la Sierra es de "cría y recría", donde los animales están a campo abierto, deambulando en campos comuneros, en los cuales en general no existen límites jurídicos formales (Ramisch et al., 2009). En la zona predomina el sistema de producción de ganadería extensiva, donde la mano de obra es familiar y el ganado vacuno pastorea junto a otros animales de crianza. En las zonas altas o de cumbre las vacas se alimentan principalmente de pastizales nativos. Mientras, en zonas de menor altitud y serranas, que se caracterizan por un aumento la diversidad vegetal, este tipo de ganadería presenta un mayor abanico de posibilidades para alimentarse. Pero en zonas de cumbre y en zonas serranas el poblador rural si bien aprovecha los recursos vegetales nativos, también recurre a especies cultivadas y forrajes comprados para complementar la alimentación de los animales.

En términos generales, los tres tipos de ganado -caprino o "majada", ovino o "rebaño" y vacuno o "hacienda"- son faenados en las unidades domésticas para el consumo familiar, siendo la carne, una parte importante de la dieta de los criollosserranos. Esta carne, también se comercializa, entre vecinos, conocidos o a carnicerías cercanas. Aunque en ocasiones se venden las crías vivas, lo común es que se entreguen faenadas para el consumo. Otro producto que se obtiene del ganado es la leche, que se bebe y se utiliza para la preparación de quesos y quesillos. Según los pobladores tan solo unas décadas atrás, también se comercializaban las pieles y las lanas, pero en la actualidad estas actividades han perdido vigencia.

En las unidades domésticas es común tener al menos un caballo (en menor medida, también se crían burros y mulas). El ganado equino, también denominado como "cabalgar" se utiliza como medio de locomoción, transporte y carga. El "caballo serrano", según los pobladores es "fuerte y resistente". $\mathrm{Al}$ igual que en otras zonas del país, los pobladores los llaman según su color de pelaje (e.j., blanco, negro, overo, tobiano). El caballo está vinculado a las actividades que generalmente en la región se consideran masculinas y en este sentido es común que sean los hombres los encargados de su cuidado. Estos animales que según los pobladores pueden llegar a vivir hasta 30 años, aún en la actualidad siguen siendo protagonistas en el campo, principalmente al momento de arar y labrar la tierra para sembrar. Sin embargo, las motos (motocicletas) siguen cobrando importancia en los espacios rurales, principalmente como un medio de locomoción en distancias cortas.

Otro tipo de animal de cría en la zona, pero que se encuentra en menor cantidad que otros tipos de ganado es el chancho o también afectuosamente conocido como el "cuchi" (Sus scrofa domestica). El chancho se cría suelto, asociado a algunos espacios domésticos como el patio (principalmente cuando son crías), aunque es más común que esté en un espacio cerrado, denominado chiquero, lugar específico para estos animales. En la región aquellos que se dedican a la cría de cerdos se les conocen como chancheros. Esta actividad según los mismos pobladores "no es para todos". Los animales al estar encerrados, precisan de ciertos cuidados, así como de aprovisionamiento de comida y de agua. En época de pariciones, los chancheros buscan pastos y pajas para elaborar en los corrales y chiqueros un tipo de cama para la chancha.

También, la cría de aves es una actividad común en Ancasti. Está presente en la mayoría de las unidades domésticas, siendo su principal fin el autoconsumo, tanto de huevos como de carne. En general, se crían gallinas (Gallus gallus domesticus), en menor medida y tan solo en algunas familias se tienen gansos (Anser spp.), patos (Anas platyrhynchos domesticus) y pavos (Meleagris gallopavo mexicana). Las gallinas se crían sueltas -sin corrales- deambulan por el terreno buscando comida y antes de la noche trepan a los árboles cercanos, a la casa o a los gallineros para dormir. 


\section{N. D. Jiménez-Escobar y G. J. Martínez - Plantas forrajeras de la Sierra de Ancasti, Catamarca}

\section{Forrajes y plantas consumidas por el ganado}

Se encontraron 154 especies, pertenecientes a 51 familias botánicas (Tabla 1). Poaceae es la familia con mayor número de especies (29), seguida de Fabaceae (13), Asteraceae (12), Bromeliaceae (10) y Cactaceae (10). En cuanto al número de géneros, se registraron 129 para Ancasti, Tillandsia presenta el mayor número de especies (6), seguido de Opuntia (4) y Setaria (4).

Los valores de riqueza de plantas forrajeras para la Sierra de Ancasti son altos en comparación con otros estudios en Argentina, que presentan: 35 especies en Chaco Seco, provincia de Santiago del Estero (Riat, 2012); 52 especies forrajeras en la región de la Pampa Seca (Muiño, 2010); 91 especies en la Puna jujeña (Quiroga Mendiola, 2011). Aun así, la riqueza para la Sierra de Ancasti resulta menor que la registrada por Scarpa (2007), quien cita 189 especies forrajeras silvestres en comunidades campesinas del norte argentino (Provincia de Formosa). Si se establecen comparaciones con comunidades campesinas en otras regiones correspondientes a ecosistemas secos en Latinoamérica: 49 especies en Bosque Seco Tropical en la zona Andina de Colombia (Galeano et al., 2013); 136 especies en Bosque Seco de la Caatinga brasilera (Nunes et al., 2015).

Según el origen geográfico de las especies, 120 especies $(77.9 \%)$ de las plantas forrajeras son nativas de la Sierra de Ancasti. Mientras, 29 especies (18.8\%), son de origen exótico. Cinco especies cuya determinación taxonómica no fue completada, son aquellas que presentan un origen desconocido (3.2\%). De acuerdo con los datos suministrados por Catálogo de plantas vasculares del Cono Sur (Zuloaga et al., 2008), seis especies forrajeras son endémicas del Cono Sur (Baccharis flabellata, Cenchrus pilcomayensis, Echinopsis aurea var. aurea, Ephedra tweediana, Lippia junelliana y Myrcianthes cisplatensis) y dos especies son endémicas de Argentina (Nassella catamarcensis y Setaria cordobensis), siendo la primera, exclusiva de áreas serranas de la Provincia de Catamarca.

En relación al hábito de las especies forrajeras, la forma herbácea es la que presenta mayor riqueza, con 86 especies $(55,8 \%$ del total), seguida por las formas arbustiva y subarbustiva $39 \mathrm{sp} .(25,3 \%)$ y arbórea $25 \mathrm{sp}$. (13,6\%). Las formas herbáceas en los ecosistemas secos cobran protagonismo por su abundancia en términos fitogeográficos referidos a la riqueza. Según Giorgis et al. (2011), el 79,5 $\%$ de las plantas del Bosque Chaqueño Serrano en la provincia de Córdoba, corresponden a la forma de vida herbácea (711 especies de 894), arbustos $(14,2 \%)$ y arboles $(6,3 \%)$. Por otro lado, las especies de porte arbustivo y arbóreo representan cerca del 39\% de las plantas forrajeras de la Sierra, esta información se debe resaltar, ya que algunos autores afirman que, para la región del Chaco Seco, es importante el rol que desempeñan árboles y arbustos en la alimentación del ganado, por el alto valor nutritivo (Sánchez et al., 2007).

Con respecto a los tipos de ganado que consumen las plantas en la Sierra, de las 154 especies registradas, las cabras consumen 124 (80,5\% del total). Un valor considerablemente superior si se compara con el número de especies que llegan a consumir otros tipos de animales de cría, como el vacuno 92 (59,7\%) o el ovino 69 (44,8\%, Fig. 2$)$.

De las 154 especies vegetales, 25 de ellas son forrajeadas exclusivamente por el ganado caprino (Tabla 1). Y es que entre los principales tipos de ganado presentes en Ancasti, se destaca el caprino, por ser el más abundante y por ser considerado como el más fácil de criar. Al igual que lo sugerido por Riat (2012), esa valoración de los cuidados de las cabras podría estar relacionado con la alta diversidad de tipos o variedades de forraje que pueden consumir. Dicho de otra forma, quizás esta menor atención en el cuidado del ganado caprino, no solo se deba a la importancia cultural y económica que le otorgan los criollos, sino también al nivel de selectividad de cada ganado respecto de la oferta natural del forraje. Si se vinculan los enunciados recientemente descritos y se relacionan con otros factores determinantes como la disponibilidad de tierras, los ingresos económicos, la capitalización de las unidades familiares, se evidencia la real importancia que tienen las cabras para el campesinado de la Sierra.

En efecto, las cabras son animales fácilmente adaptables; varios autores han sugerido que, debido a las características anatómicas-fisiológicas, pueden adaptar su dieta de acuerdo al tipo de forraje disponible, variando la composición alimentaria según la calidad y la oferta, pasando de ser consumidoras generalistas a especialistas, en la medida que el ambiente alimenticio mejore (Roig, 2003). Asimismo, De Rancourt et al. (2006) afirman que entre las ventajas del ganado rumiante (caprino 


\section{Tabla 1. Lista comentada de las especies forrajeras de la Sierra de Ancasti, Catamarca.}

\begin{tabular}{|c|c|c|c|c|c|c|}
\hline FAMILIA, Especie, Vaucher & Nombre común & Origen & Hábitat & $\mathrm{Fr} \%$ & Parte & Forraje \\
\hline \multicolumn{7}{|l|}{ AMARANTHACEAE } \\
\hline $\begin{array}{l}\text { Amaranthus hybridus L. ssp. } \\
\text { hybridus, NDJ } 2308\end{array}$ & ataco & $E$ & $\mathrm{Pe}$ & 15 & $\mathrm{~Pa}$ & ca, ov, po \\
\hline Amaranthus cf. spinosus L., GJM 931 & ataco & $\mathrm{N}$ & $\mathrm{Pe}$ & 10 & $\mathrm{~Pa}$ & ca, ov, po \\
\hline Amaranthus sp.1 GJM 1433 & ataco & SD & $\mathrm{Pe}$ & 10 & $\mathrm{~Pa}$ & ca, ov, po \\
\hline Gomphrena haenkeana Mart. NDJ 2315 & SN & $\mathrm{N}$ & $\mathrm{Cu}, \mathrm{Si}$ & 10 & $\mathrm{~Pa}$ & ca, ov \\
\hline \multicolumn{7}{|l|}{ ANACARDIACEAE } \\
\hline Lithraea molleoides (Vell.) Engl. GJM 883 & molle, molle de beber & $\mathrm{N}$ & Si & 25 & $\mathrm{Fr}, \mathrm{Ho}$ & ca, ov, va \\
\hline $\begin{array}{l}\text { Schinus fasciculatus (Griseb.) I.M. } \\
\text { Johnst. var. fasciculatus GJM } 1263\end{array}$ & molle tonto, molle pispo & $\mathrm{N}$ & $\mathrm{Si}$ & 30 & $\mathrm{Fr}, \mathrm{Ho}$ & ca, ov \\
\hline \multicolumn{7}{|l|}{ APIACEAE } \\
\hline Coriandrum sativum L., NDJ 2016 & cilantro & $E$ & $\mathrm{Pe}$ & 10 & $\mathrm{~Pa}$ & ca \\
\hline Eryngium ebracteatum Lam., NDJ 2233 & escorcera & $\mathrm{N}$ & $\mathrm{Cu}, \mathrm{Si}$ & 15 & $\mathrm{Ho}, \mathrm{Ra}$ & po, va \\
\hline Hydrocotyle ranunculoides L. f., NDJ 2015 & $\begin{array}{l}\text { redondo del agua, } \\
\text { arandela del agua }\end{array}$ & $\mathrm{N}$ & Si & 20 & $\mathrm{~Pa}$ & $\begin{array}{l}\text { ca, eq, } \\
\text { ov, va }\end{array}$ \\
\hline \multicolumn{7}{|l|}{ ARACEAE } \\
\hline Lemma gibba L., NDJ 2016 & rojilla & $\mathrm{N}$ & $\mathrm{Si}$ & 10 & PE & va \\
\hline \multicolumn{7}{|l|}{ ASPARAGACEAE } \\
\hline Agave americana L., NDJ 2340 & pita & $E$ & $\mathrm{Pe}$ & 10 & $\mathrm{~Pa}$ & va \\
\hline \multicolumn{7}{|l|}{ ASTERACEAE } \\
\hline Acanthospermum hispidum DC., NDJ 2292 & horquetilla & $\mathrm{N}$ & $\mathrm{Si}$ & 10 & $\mathrm{~Pa}$ & ca, ov \\
\hline $\begin{array}{l}\text { Achyrocline satureioides } \\
\text { (Lam.) DC., GJM } 1284\end{array}$ & salvia & $\mathrm{N}$ & Si & 10 & $\mathrm{~Pa}$ & ca \\
\hline Baccharis flabellata Hook. \& Arn., NDJ 2245 & clavillo & $\mathrm{N}$ & $\mathrm{Cu}$ & 10 & $\mathrm{Ho}$ & ca, ov, va \\
\hline Baccharis ulicina Hook. \& Arn. NDJ 2268 & $\begin{array}{l}\text { yerba meona, } \\
\text { yerba oveja }\end{array}$ & $\mathrm{N}$ & $\mathrm{Cu}, \mathrm{Si}$ & 10 & $\mathrm{~Pa}$ & ov \\
\hline $\begin{array}{l}\text { Gaillardia megapotamica (Spreng.) } \\
\text { Baker var. scabiosoides (Arn. ex DC.) } \\
\text { Baker, GJM 862, 1149; NDJ } 2254\end{array}$ & botón de oro, topasaire & $\mathrm{N}$ & Si & 10 & $\mathrm{~Pa}$ & ca, ov \\
\hline Helianthus annuus L., SC & girasol & A & VC & 10 & $\mathrm{Se}$ & av \\
\hline $\begin{array}{l}\text { Parthenium hysterophorus L. } \\
\text { GJM 1199; NDJ } 2291\end{array}$ & altamisa & $\mathrm{N}$ & $\mathrm{Si}$ & 10 & $\mathrm{~Pa}$ & $\mathrm{ca}$ \\
\hline Pectis odorata Griseb., NDJ 2320 & manzanilla dulce & $\mathrm{N}$ & $\mathrm{Si}$ & 10 & $\mathrm{~Pa}$ & ca, ov \\
\hline Pseudognaphalium sp.1, NDJ 2311 & salvia blanca & $\mathrm{N}$ & $\mathrm{Cu}, \mathrm{Si}$ & 10 & $\mathrm{~Pa}$ & ca, ov \\
\hline $\begin{array}{l}\text { Schkuhria pinnata (Lam.) Kuntze } \\
\text { ex Thell., GJM } 860,1280\end{array}$ & $\begin{array}{l}\text { matapulgas, } \\
\text { canchalagua }\end{array}$ & $\mathrm{N}$ & $\mathrm{Cu}, \mathrm{Si}$ & 10 & $\mathrm{~Pa}$ & va \\
\hline $\begin{array}{l}\text { Xanthium spinosum L. var. } \\
\text { spinosum, GJM 904; NDJ } 2269\end{array}$ & cepacaballo & A & $\mathrm{Cu}, \mathrm{Si}$ & 15 & $\mathrm{Ho}$ & $\mathrm{eq}^{*}, \mathrm{va}$ \\
\hline Zinnia peruviana (L.) L., GJM 872; NDJ 2248 & chinita & $\mathrm{N}$ & $\mathrm{Cu}, \mathrm{Si}$ & 20 & $\mathrm{~Pa}$ & ca, eq, ov \\
\hline \multicolumn{7}{|l|}{ AZOLLACEAE } \\
\hline Azolla filiculoides Lam., NDJ 2022 & cresta de gallo & $\mathrm{N}$ & $\mathrm{Si}$ & 20 & PE & va \\
\hline \multicolumn{7}{|l|}{ BRASSICACEAE } \\
\hline Nasturtium officinale W.T. Aiton, SC & berro & A & Si & 15 & $\mathrm{~Pa}$ & $\begin{array}{l}\text { av, ca, } \\
\text { po, va }\end{array}$ \\
\hline \multicolumn{7}{|l|}{ BROMELIACEAE } \\
\hline $\begin{array}{l}\text { Deuterocohnia longipetala } \\
\text { (Baker) Mez, NDJ } 2322\end{array}$ & chaguar & $\mathrm{N}$ & $\mathrm{Cu}, \mathrm{Si}$ & 30 & Ho & ca, va \\
\hline Dickya floribunda Griseb., SC & chaguar & $\mathrm{N}$ & $\mathrm{Si}$ & 30 & Ho & ca, va \\
\hline Puya spathacea (Griseb.) Mez, NDJ 1992 & chaguar & $\mathrm{N}$ & & 35 & $\mathrm{Ho}$ & $\mathrm{ca}, \mathrm{eq}^{*}, \mathrm{va}$ \\
\hline $\begin{array}{l}\text { Tillandsia argentina C. H. } \\
\text { Wright, NDJ 2000, } 2285\end{array}$ & azahar & $\mathrm{N}$ & $\mathrm{Cu}, \mathrm{Si}$ & 35 & PE & ca, ov, va \\
\hline Tillandsia capillaris Ruiz \& Pav., NDJ 2010 & clavel del aire & $\mathrm{N}$ & $\mathrm{Si}$ & 30 & PE & ca, eq, va \\
\hline Tillandsia duratii Vis., NDJ 2021, 2288 & azahar & $\mathrm{N}$ & $\mathrm{Si}$ & 35 & $\mathrm{PE}$ & ca, po, va \\
\hline
\end{tabular}


Continuación Tabla 1

\begin{tabular}{|c|c|c|c|c|c|c|}
\hline FAMILIA, Especie, Vaucher & Nombre común & Origen & Hábitat & Fr\%* & Parte & Forraje \\
\hline Tillandsia ixioides Griseb., NDJ 1983 & azahar & $\mathrm{N}$ & $\mathrm{Cu}, \mathrm{Si}$ & 35 & PE & $\begin{array}{l}\text { ca, eq, } \\
\text { ov, va }\end{array}$ \\
\hline Tillandsia lorentziana Griseb., GJM 1050 & azahar & $\mathrm{N}$ & $\mathrm{Si}$ & 35 & PE & ca, eq, va \\
\hline Tillandsia minutiflora Donadío, NDJ 2017 & $\begin{array}{l}\text { clavel del aire, } \\
\text { flor del aire }\end{array}$ & $\mathrm{N}$ & $\mathrm{Si}$ & 30 & PE & ca, va \\
\hline Tillandsia xiphioides Ker Gawl., NDJ 1983 & azahar & $\mathrm{N}$ & $\mathrm{Si}$ & 35 & PE & $\begin{array}{l}\text { ca, eq, } \\
\text { ov, va }\end{array}$ \\
\hline \multicolumn{7}{|l|}{ CACTACEAE } \\
\hline Cereus forbesii Otto ex C.F. Först. NDJ 2300 & ucle, cacto de san Juan & $\mathrm{N}$ & $\mathrm{Si}$ & 55 & $\mathrm{~Pa}$ & ca, ov, va \\
\hline $\begin{array}{l}\text { Cleistocactus smaragdiflorus (F.A.C. } \\
\text { Weber) Britton \& Rose, SC }\end{array}$ & alaba, cola de zorro & $\mathrm{N}$ & Si & 25 & $\mathrm{Ta}$ & ca, ov, va \\
\hline $\begin{array}{l}\text { Cleitocactus cf. baumannii } \\
\text { (Lem.) Lem. GJM } 1088\end{array}$ & $\begin{array}{l}\text { bola de gato, } \\
\text { huevo de gato }\end{array}$ & $\mathrm{N}$ & $\mathrm{Si}$ & 10 & $\mathrm{~Pa}$ & po \\
\hline $\begin{array}{l}\text { Echinopsis aurea Britton \& Rose } \\
\text { var. aurea, NDJ } 2280\end{array}$ & $\begin{array}{l}\text { machocorota, } \\
\text { machocorote }\end{array}$ & $\mathrm{N}$ & $\mathrm{Cu}, \mathrm{Si}$ & 15 & $\mathrm{~Pa}$ & ca, ov \\
\hline $\begin{array}{l}\text { Harrisia pomanensis (F.A.C. Weber } \\
\text { ex K. Schum.) Britton \& Rose ssp. } \\
\text { pomanensis, GJM 1034B, } 1104\end{array}$ & ulva & $\mathrm{N}$ & $\mathrm{Si}$ & 20 & $\mathrm{~Pa}, \mathrm{FI}$ & ca, po \\
\hline $\begin{array}{l}\text { Opuntia ficus-indica (L.) } \\
\text { Mill., GJM 1168, } 1426\end{array}$ & tuna & A & $\mathrm{Pe}$ & 10 & $\mathrm{Ta}$ & va \\
\hline Opuntia prasina Speg., NDJ 2279 & penca belenista & SD & $\mathrm{Pe}$ & 10 & $\mathrm{~Pa}$ & ca, ov, va \\
\hline Opuntia salmiana Parm., NDJ 2296 & cola de león & $\mathrm{N}$ & $\mathrm{Si}$ & 10 & $\mathrm{Fr}$ & ca, po \\
\hline $\begin{array}{l}\text { Opuntia sulphurea Gilles ex Salm-Dyck } \\
\text { var. pampeana (Speg.) Backeb., SC }\end{array}$ & quishcaloro, quiscaludo & $\mathrm{N}$ & $\mathrm{Si}$ & 30 & $\mathrm{~Pa}, \mathrm{Fr}$ & ca, ov, va \\
\hline $\begin{array}{l}\text { Trichocereus terscheckii (Parm. ex } \\
\text { Pfeiff.) Britton \& Rose, NDJ } 2301\end{array}$ & achuma, cardón & $\mathrm{N}$ & $\mathrm{Cu}, \mathrm{Si}$ & 30 & $\mathrm{Ta}$ & ca, va \\
\hline \multicolumn{7}{|l|}{ CANNACEAE } \\
\hline Canna indica L., SC & achera & $\mathrm{N}$ & $\mathrm{Pe}$ & 10 & Ho, Ra & po \\
\hline \multicolumn{7}{|l|}{ CARYOPHYLLACEAE } \\
\hline $\begin{array}{l}\text { Polycarpon suffruticosum Griseb., } \\
\text { NDJ 1993, 2246, } 2307\end{array}$ & $\begin{array}{l}\text { helecho chinito, } \\
\text { helecho del campo }\end{array}$ & $\mathrm{E}$ & Si & 20 & $\mathrm{~Pa}$ & ca, ov \\
\hline \multicolumn{7}{|l|}{ CELTIDACEAE } \\
\hline $\begin{array}{l}\text { Celtis ehrenbergiana (Klotzsch) } \\
\text { Liebm., GJM 905; NDJ } 2295\end{array}$ & tala, tala pispito & $\mathrm{N}$ & $\mathrm{Si}$ & 15 & $\mathrm{~Pa}$ & $\begin{array}{l}\text { av, ca, } \\
\text { ov, po }\end{array}$ \\
\hline $\begin{array}{l}\text { Celtis iguanaea (Jacq.) Sarg., } \\
\text { GJM 1055, 1371; NDJ } 2249\end{array}$ & $\begin{array}{l}\text { horco tala, tala, } \\
\text { tala macho }\end{array}$ & $\mathrm{N}$ & $\mathrm{Si}$ & 15 & $\mathrm{~Pa}$ & ca, ov, va \\
\hline \multicolumn{7}{|l|}{ CHENOPODIACEAE } \\
\hline $\begin{array}{l}\text { Dysphania ambrosioides (L.) } \\
\text { Mosyakin \& Clemants, GJM } 947\end{array}$ & paico & $\mathrm{N}$ & $\mathrm{Pe}$ & 10 & $\mathrm{~Pa}$ & ca \\
\hline \multicolumn{7}{|l|}{ CONVOLVULACEAE } \\
\hline Ipomoea purpurea (L.) Roth, NDJ 2232 & $\begin{array}{l}\text { porotillo, suspiro } \\
\text { (flor violeta) }\end{array}$ & $\mathrm{N}$ & $\mathrm{Pe}$ & 20 & PE & $\begin{array}{l}\text { ca, eq, } \\
\text { po, ov }\end{array}$ \\
\hline Ipomoea sp. SC & porotillo (flor blanca) & SD & $\mathrm{Pe}$ & 15 & PE & ca, ov, po \\
\hline \multicolumn{7}{|l|}{ CUCURBITACEAE } \\
\hline Cucurbita maxima Duch., SC & zapallo & $\mathrm{E}$ & $\mathrm{Pe}$ & 15 & $\mathrm{Fr}$ & ca, po, va \\
\hline \multicolumn{7}{|l|}{ CYPERACEAE } \\
\hline Cyperus corymbosus Rottb., NDJ 1991 & pasto colorado & $\mathrm{N}$ & $\mathrm{Si}$ & 10 & $\mathrm{~Pa}$ & $\begin{array}{l}\text { ca, eq, } \\
\text { ov, va }\end{array}$ \\
\hline Cyperus haspan L., NDJ 2236 & pasto "natural" & $\mathrm{N}$ & $\mathrm{Si}$ & 10 & $\mathrm{~Pa}$ & $\begin{array}{l}\text { ca, eq, } \\
\text { ov, va }\end{array}$ \\
\hline $\begin{array}{l}\text { Cyperus esculentus L. } \\
\text { esculentus L., NDJ } 2314\end{array}$ & pasto & $E$ & $\mathrm{Cu}, \mathrm{Si}$ & 10 & $\mathrm{~Pa}$ & eq, va \\
\hline Cyperus niger Ruiz \& Pav., NDJ 2251 & paja & $\mathrm{N}$ & $\mathrm{Cu}$ & 10 & $\mathrm{~Pa}$ & eq, ov, va \\
\hline $\begin{array}{l}\text { Rhynchospora cf. rugosa } \\
\text { (Vahl) Gale, NDJ } 2273\end{array}$ & pasto & $\mathrm{N}$ & $\mathrm{Si}$ & 15 & $\mathrm{~Pa}$ & ca, eq, ov \\
\hline
\end{tabular}


Continuación Tabla 1

\begin{tabular}{|c|c|c|c|c|c|c|}
\hline FAMILIA, Especie, Vaucher & Nombre común & Origen & Hábitat & $\mathrm{Fr} \%$ * & Parte & Forraje \\
\hline \multicolumn{7}{|l|}{ EPHEDRACEAE } \\
\hline $\begin{array}{l}\text { Ephedra tweediana Fisch. \& } \\
\text { C.A. Mey. emend. J.H. Hunz., } \\
\text { GJM 1035, 1170; NDJ } 1987\end{array}$ & tramontana & $\mathrm{N}$ & $\mathrm{Cu}$ & 45 & $\mathrm{~Pa}$ & $\begin{array}{l}\text { eq*, ca, } \\
\text { ov, va }\end{array}$ \\
\hline \multicolumn{7}{|l|}{ EUPHORBIACEAE } \\
\hline $\begin{array}{l}\text { Cnidoscolus tubulosus (Müll. Arg.) } \\
\text { I.M. Johnst. var. trilobus (Müll. Arg.) } \\
\text { Lourteig \& O’Donell, GJM } 885\end{array}$ & ya te veo & $\mathrm{N}$ & $\mathrm{Si}$ & 25 & $\mathrm{Ra}$ & po \\
\hline $\begin{array}{l}\text { Croton lachnostachyus Baill., } \\
\text { GJM 856, 894; NDJ } 2256\end{array}$ & tinajera & $\mathrm{N}$ & $\mathrm{Cu}, \mathrm{Si}$ & 10 & $\mathrm{~Pa}$ & ca, va \\
\hline $\begin{array}{l}\text { Euphorbia hirta L. var. ophthalmica } \\
\text { (Pers.) Allem \& Irgang, NDJ } 2312\end{array}$ & hierba de golondrina & $E$ & $\mathrm{Si}$ & 10 & $\mathrm{~Pa}$ & $\mathrm{ca}$ \\
\hline Ricinus communis L. & tártago & $E$ & $\mathrm{Pe}$ & 10 & Ho & ca \\
\hline \multicolumn{7}{|l|}{ FABACEAE } \\
\hline $\begin{array}{l}\text { Adesmia muricata (Jacq.) DC. var. } \\
\text { dentata (Lag.) Benth., NDJ } 2004\end{array}$ & boca de pato & $\mathrm{N}$ & $\mathrm{Si}$ & 10 & $\mathrm{~Pa}$ & $\begin{array}{l}\text { ca, eq, } \\
\text { ov, va }\end{array}$ \\
\hline $\begin{array}{l}\text { Anadenanthera colubrina (Vell.) Brenan var. } \\
\text { cebil (Griseb.) Altschul, GJM 925, } 1191\end{array}$ & cébil & $\mathrm{N}$ & $\mathrm{Si}$ & 15 & $\mathrm{Fr}, \mathrm{Ho}$ & ca, ov, va \\
\hline $\begin{array}{l}\text { Geoffroea decorticans (Gillies ex } \\
\text { Hook. \& Arn.) Burkart, NDJ } 2219\end{array}$ & chañar & $\mathrm{N}$ & $\mathrm{Si}$ & 40 & $\mathrm{Fr}$ & ca, po, va \\
\hline Medicago sativa L., SC & alfalfa, alfa & $E$ & VC & 75 & $\mathrm{~Pa}$ & eq, va \\
\hline $\begin{array}{l}\text { Mimosa farinosa Griseb. GJM } \\
889,1087 \text {; NDJ } 2023\end{array}$ & shinqui & $\mathrm{N}$ & $\mathrm{Cu}, \mathrm{Si}$ & 45 & $\begin{array}{l}\mathrm{Pa} \text {, } \\
\mathrm{FI}, \mathrm{Fr}\end{array}$ & $\begin{array}{l}\text { eq*, ca, } \\
\text { ov, va }\end{array}$ \\
\hline $\begin{array}{l}\text { Parapiptadenia excelsa (Griseb.) } \\
\text { Burkart, GJM 1057, 1256, } 1326\end{array}$ & viscote colorado & $\mathrm{N}$ & $\mathrm{Si}$ & 20 & $\mathrm{Fr}$, Ho & ca, va \\
\hline $\begin{array}{l}\text { Parasenegalia visco (Lorentz ex Griseb.) } \\
\text { Seigler \& Ebinge, GJM 897, 1053, NDJ } 2277\end{array}$ & viscote, viscote negro & $\mathrm{N}$ & $\mathrm{Si}$ & 45 & Fr, Ho & $\begin{array}{l}\text { ca, eq, } \\
\text { ov, va }\end{array}$ \\
\hline Prosopis alba Griseb., GJM 1223 & algarrobo & $\mathrm{N}$ & $\mathrm{Si}$ & 30 & $\mathrm{Fr}$ & $\begin{array}{l}\text { ca, eq, } \\
\text { ov, va }\end{array}$ \\
\hline Prosopis nigra (Griseb.) Hieron., GJM 949 & algarrobo negro & $\mathrm{N}$ & $\mathrm{Si}$ & 10 & Fr & ca, va \\
\hline $\begin{array}{l}\text { Prosopis sericantha Gillies ex } \\
\text { Hook. \& Arn., GJM 1217, } 1229\end{array}$ & albardón & $\mathrm{N}$ & $\mathrm{Si}$ & 10 & $\mathrm{Fr}$ & $\mathrm{ca}$ \\
\hline Robinia pseudoacacia L., NDJ 2361, 2386 & acacio & $\mathrm{E}$ & $\mathrm{Pe}$ & 10 & $\mathrm{Fr}, \mathrm{Ho}$ & ca \\
\hline $\begin{array}{l}\text { Vachellia aroma (Gillies ex Hook. } \\
\text { \& Arn.) Seigler \& Ebinger, GJM } \\
870,1298 ; \text { NDJ } 2014\end{array}$ & $\begin{array}{l}\text { tusquilla, algarrobilla, } \\
\text { tusca }\end{array}$ & $\mathrm{N}$ & $\mathrm{Cu}, \mathrm{Si}$ & 50 & $\begin{array}{l}\mathrm{FI}, \mathrm{Fr} \\
\mathrm{Ho}\end{array}$ & ca, ov, va \\
\hline $\begin{array}{l}\text { Vachellia caven (Molina) Seigler } \\
\text { \& Ebinger, GJM } 871,1297\end{array}$ & churqui, tusca & $\mathrm{N}$ & $\mathrm{Cu}, \mathrm{Si}$ & 65 & $\begin{array}{l}\mathrm{FI}, \mathrm{Fr} \\
\mathrm{Ho}\end{array}$ & $\begin{array}{l}\text { ca, eq, } \\
\text { ov, va }\end{array}$ \\
\hline \multicolumn{7}{|l|}{ JUGLANDACEAE } \\
\hline $\begin{array}{l}\text { Juglans australis Griseb. } \\
\text { GJM 1046, NDJ } 2020\end{array}$ & nogal cimarrón & $\mathrm{N}$ & $\mathrm{Pe}$ & 30 & $\mathrm{Fr}$ & ca, po, va \\
\hline \multicolumn{7}{|l|}{ LAMIACEAE } \\
\hline Hedeoma multiflora Benth., GJM 1257 & tomillo & $\mathrm{N}$ & $\mathrm{Si}$ & 15 & $\mathrm{~Pa}$ & ov \\
\hline \multicolumn{7}{|l|}{ LORANTHACEAE } \\
\hline $\begin{array}{l}\text { Ligaria cuneifolia (Ruiz \& Pav.) } \\
\text { Tiegh., GJM 1283, NDJ } 2281\end{array}$ & liga & $\mathrm{N}$ & $\mathrm{Si}$ & 35 & PE & ca, ov, va \\
\hline $\begin{array}{l}\text { Struthanthus uraguensis (Hook. } \\
\text { \& Arn.) G. Don, GJM } 1180\end{array}$ & liga & $\mathrm{N}$ & $\mathrm{Si}$ & 35 & PE & ca, ov, va \\
\hline $\begin{array}{l}\text { Tripodanthus acutifolius (Ruiz \& Pav.) } \\
\text { Tiegh., GJM 957, 1264; NDJ 2001, } 2284\end{array}$ & corpo, corpus & $\mathrm{N}$ & $\mathrm{Si}$ & 15 & PE & ca \\
\hline \multicolumn{7}{|l|}{ LYTHRACEAE } \\
\hline $\begin{array}{l}\text { Heimia salicifolia (Kunth) Link, } \\
\text { GJM } 855 \text {, NDJ } 2258\end{array}$ & arupaco, quiebraarado & $\mathrm{N}$ & $\mathrm{Cu}, \mathrm{Si}$ & 15 & $\mathrm{~Pa}$ & ca, ov \\
\hline \multicolumn{7}{|l|}{ MALPIGHIACEAE } \\
\hline $\begin{array}{l}\text { Heteropterys dumetorum } \\
\text { (Griseb.) Nied., GJM } 1202\end{array}$ & loconte amarillo & $\mathrm{N}$ & $\mathrm{Si}$ & 10 & $\mathrm{~Pa}$ & ca \\
\hline
\end{tabular}


Continuación Tabla 1

\begin{tabular}{|c|c|c|c|c|c|c|}
\hline FAMILIA, Especie, Vaucher & Nombre común & Origen & Hábitat & Fr\%* & Parte & Forraje \\
\hline \multicolumn{7}{|l|}{ MALVACEAE } \\
\hline $\begin{array}{l}\text { Ceiba chodatii (Hassl.) Ravenna, } \\
\text { GJM 1192; NDJ } 2296\end{array}$ & palo borracho & $\mathrm{N}$ & $\mathrm{Si}$ & 25 & Ho, Ta & ca, ov, va \\
\hline $\begin{array}{l}\text { Sphaeralcea bonariensis } \\
\text { (Cav.) Griseb., GJM } 854\end{array}$ & malva, malva blanca & $\mathrm{N}$ & $\mathrm{Si}$ & 10 & $\mathrm{Ho}$ & ca, po, va \\
\hline \multicolumn{7}{|l|}{ MORACEAE } \\
\hline $\begin{array}{l}\text { Broussonetia papyrifera (L.) L'Hér. } \\
\text { ex Vent., GJM 1243, NDJ } 2287\end{array}$ & gomero, mora turca & $\mathrm{E}$ & $\mathrm{Pe}$ & 15 & $\mathrm{Ho}$ & eq, va \\
\hline Morus alba L., SC & mora, mora criolla & $E$ & $\mathrm{Pe}$ & 20 & Ho & ca, va \\
\hline \multicolumn{7}{|l|}{ MYRTACEAE } \\
\hline $\begin{array}{l}\text { Myrcianthes cisplatensis (Cambess.) } \\
\text { O. Berg, GJM 895, 1044, } 1173\end{array}$ & huil, mato huil & $\mathrm{N}$ & Si & 45 & $\mathrm{~Pa}$ & ca, ov, va \\
\hline \multicolumn{7}{|l|}{ NYCTAGINACEAE } \\
\hline Bougainvillea stipitata Griseb., GJM 896 & espinillo & $\mathrm{N}$ & $\mathrm{Si}$ & 15 & $\mathrm{Ho}$ & ca \\
\hline \multicolumn{7}{|l|}{ OLEACEAE } \\
\hline Ligustrum lucidum W.T. Aiton, NDJ 2370 & siempre verde & $E$ & $\mathrm{Pe}$ & 10 & $\mathrm{Ho}$ & ca \\
\hline \multicolumn{7}{|l|}{ ORCHIDACEAE } \\
\hline $\begin{array}{l}\text { Oncidium bifolium Sims var. } \\
\text { majus Hort, NDJ } 2299\end{array}$ & margarita & $\mathrm{N}$ & $\mathrm{Si}$ & 15 & $\mathrm{~Pa}$ & ca, va \\
\hline \multicolumn{7}{|l|}{ OXALIDACEAE } \\
\hline $\begin{array}{l}\text { Oxalis conorrhiza Jacq., } \\
\text { GJM 1258; NDJ } 2252\end{array}$ & saladillo, vinagrillo & $\mathrm{N}$ & $\mathrm{Cu}, \mathrm{Si}$ & 15 & $\mathrm{~Pa}$ & ca, ov \\
\hline \multicolumn{7}{|l|}{ PARMELIACEAE } \\
\hline $\begin{array}{l}\text { Usnea cf. barbata (L.) Weber ex F.H. } \\
\text { Wigg. GJM 1094, 1251, } 1370\end{array}$ & $\begin{array}{l}\text { barba de la peña, } \\
\text { barba de monte }\end{array}$ & $\mathrm{N}$ & $\mathrm{Cu}, \mathrm{Si}$ & 15 & PE & $\mathrm{ca}$ \\
\hline \multicolumn{7}{|l|}{ PLANTAGINACEAE } \\
\hline $\begin{array}{l}\text { Linaria canadensis (L.) Dum. } \\
\text { Cours., NDJ } 2005\end{array}$ & clavelina & $\mathrm{E}$ & $\mathrm{Si}$ & 10 & $\mathrm{~Pa}$ & ca \\
\hline \multicolumn{7}{|l|}{ POACEAE } \\
\hline Aristida adscensionis L., NDJ 2293, 2319 & pasto, pasto flecha & $\mathrm{N}$ & $\mathrm{Cu}, \mathrm{Si}$ & 10 & $\mathrm{~Pa}$ & ca, va \\
\hline Avena spp., SC & avena & $E$ & VC & 15 & $\mathrm{Fr}$ & eq \\
\hline $\begin{array}{l}\text { Bothriochloa springfieldii } \\
\text { (Gould) Parodi, GJM } 1271\end{array}$ & pasto & $\mathrm{N}$ & $\mathrm{Cu}, \mathrm{Si}$ & 10 & $\mathrm{~Pa}$ & $\begin{array}{l}\text { ca, eq, } \\
\text { ov, va }\end{array}$ \\
\hline $\begin{array}{l}\text { Bouteloua megapotamica } \\
\text { (Spreng.) Kuntze, NDJ } 2289\end{array}$ & pasto, paja fina & $\mathrm{N}$ & $\mathrm{Cu}, \mathrm{Si}$ & 15 & $\mathrm{~Pa}$ & $\begin{array}{l}\text { ca, eq, } \\
\text { ov, va }\end{array}$ \\
\hline $\begin{array}{l}\text { Cenchrus pilcomayensis (Mez) } \\
\text { Morrone, NDJ } 2259\end{array}$ & pasto simbol & $\mathrm{N}$ & $\mathrm{Cu}, \mathrm{Si}$ & 20 & $\mathrm{~Pa}$ & ca, ov, va \\
\hline $\begin{array}{l}\text { Cenchrus spinifex Cav., } \\
\text { GJM 1269; NDJ } 2283\end{array}$ & roseta & $\mathrm{N}$ & $\mathrm{Cu}, \mathrm{Si}$ & 15 & $\mathrm{~Pa}$ & ca, ov, va \\
\hline $\begin{array}{l}\text { Cortaderia selloana (Schult. \& Schult. } \\
\text { f.) Asch. \& Graebn., NDJ } 2242\end{array}$ & cortadera & $\mathrm{N}$ & $\mathrm{Cu}, \mathrm{Si}$ & 15 & $\mathrm{~Pa}$ & ca, va \\
\hline $\begin{array}{l}\text { Cynodon dactylon (L.) Pers., } \\
\text { NDJ } 2230,2310\end{array}$ & grama & A & $\mathrm{Cu}, \mathrm{Si}$ & 25 & $\mathrm{~Pa}$ & $\begin{array}{l}\text { ca, eq, } \\
\text { ov, va }\end{array}$ \\
\hline $\begin{array}{l}\text { Disakisperma dubium (Kunth) P.M. } \\
\text { Peterson \& N.W. Snow, NDJ } 2321\end{array}$ & pasto & $\mathrm{N}$ & $\mathrm{Cu}$ & 10 & $\mathrm{~Pa}$ & ca \\
\hline $\begin{array}{l}\text { Eragrostis orthoclada Hack., } \\
\text { GJM 1275; NDJ 2305, } 2317\end{array}$ & pasto/pastura & $\mathrm{N}$ & $\mathrm{Cu}$ & 15 & $\mathrm{~Pa}$ & ca, ov, va \\
\hline Eustachys retusa (Lag.) Kunth, NDJ 2278 & pasto "tipo"gramilla & $\mathrm{N}$ & $\mathrm{Cu}, \mathrm{Si}$ & 15 & $\mathrm{~Pa}$ & eq, va \\
\hline Hordeum spp., SC & cebada & A & VC & 20 & $\mathrm{Fr}$ & eq \\
\hline $\begin{array}{l}\text { Jarava ichu Ruiz \& Pav. } \\
\text { var. Ichu, NDJ 2228, } 2316\end{array}$ & $\begin{array}{l}\text { paja brava, paja } \\
\text { dura, paja gruesa }\end{array}$ & $\mathrm{N}$ & $\mathrm{Cu}, \mathrm{Si}$ & 25 & $\mathrm{~Pa}$ & ca, eq, va \\
\hline $\begin{array}{l}\text { Jarava leptostachya (Griseb.) } \\
\text { F. Rojas, NDJ } 2306\end{array}$ & Paja, paja cumbrera & $\mathrm{N}$ & $\mathrm{Cu}, \mathrm{Si}$ & 35 & $\mathrm{~Pa}$ & $\begin{array}{l}\text { ca, eq, } \\
\text { ov, va }\end{array}$ \\
\hline $\begin{array}{l}\text { Leptochloa fusca (L.) Kunth ssp. } \\
\text { fascicularis (Lam.) N.W. Snow, GJM } 2241\end{array}$ & pasto "natural" & $\mathrm{N}$ & $\mathrm{Cu}, \mathrm{Si}$ & 10 & $\mathrm{~Pa}$ & eq \\
\hline Nassella catamarcensis Torres, NDJ 2018 & cebadilla & $\mathrm{N}$ & $\mathrm{Cu}$ & 15 & $\mathrm{~Pa}$ & ca, ov, va \\
\hline Paspalum malacophyllum Trin., NDJ 2274 & pasto & $\mathrm{N}$ & $\mathrm{Cu}, \mathrm{Si}$ & 10 & $\mathrm{~Pa}$ & ca, ov, va \\
\hline
\end{tabular}


Continuación Tabla 1

\begin{tabular}{|c|c|c|c|c|c|c|}
\hline FAMILIA, Especie, Vaucher & Nombre común & Origen & Hábitat & $\mathrm{Fr} \%$ * & Parte & Forraje \\
\hline $\begin{array}{l}\text { Paspalum notatum Flüggé var. notatum, } \\
\text { GJM 1276; NDJ 2234, } 2282\end{array}$ & $\begin{array}{l}\text { grama colorada, } \\
\text { gramilla, pasto }\end{array}$ & $\mathrm{N}$ & $\mathrm{Cu}, \mathrm{Si}$ & 45 & $\mathrm{~Pa}$ & ca, eq, va \\
\hline Poa calchaquiensis Hack., NDJ 2272 & pasto & $\mathrm{N}$ & $\mathrm{Cu}$ & 15 & $\mathrm{~Pa}$ & $\mathrm{ca}$ \\
\hline $\begin{array}{l}\text { Setaria cordobensis R.A.W. } \\
\text { Herrm. GJM } 1272\end{array}$ & pasto & $\mathrm{N}$ & $\mathrm{Si}$ & 10 & $\mathrm{~Pa}$ & ca, eq, va \\
\hline Setaria macrostachya Kunth & cadillo & $\mathrm{N}$ & $\mathrm{Cu}, \mathrm{Si}$ & 15 & $\mathrm{~Pa}$ & $\begin{array}{l}\text { ca, eq, } \\
\text { ov, va }\end{array}$ \\
\hline $\begin{array}{l}\text { Setaria parviflora (Poir.) Kerguelén } \\
\text { var. parviflora, NDJ } 2240\end{array}$ & pasto "natural" & $\mathrm{N}$ & $\mathrm{Cu}, \mathrm{Si}$ & 15 & $\mathrm{~Pa}$ & ca, eq \\
\hline Setaria sp.1, GJM 1270 & pasto & SD & $\mathrm{Cu}$ & 10 & $\mathrm{~Pa}$ & ca, eq, va \\
\hline Sorghum halepense (L.) Pers., GJM 1171 & pasto ruso & A & $\mathrm{Cu}, \mathrm{Si}$ & 20 & $\mathrm{~Pa}$ & eq, va \\
\hline Sorghum spp. SC & pasto sorgo & A & $\mathrm{Cu}, \mathrm{Si}$ & 20 & $\mathrm{~Pa}$ & eq, va \\
\hline Tragus berteronianus Schult., GJM 1174 & pasto & $A$ & $\mathrm{Si}$ & 10 & $\mathrm{~Pa}$ & ca, ov, va \\
\hline Triticum spp., SC & trigo & A & VC & 25 & $\mathrm{~Pa}$ & ca, eq, va \\
\hline Zea mays L., SC & maíz & A & $\mathrm{Pe}, \mathrm{VC}$ & 60 & $\begin{array}{l}\mathrm{Fr}, \mathrm{Ho} \\
\mathrm{Se}\end{array}$ & $\begin{array}{l}\text { av, ca, eq, } \\
\text { ov, po, va }\end{array}$ \\
\hline POACEAE sp.1, GJM 1268 & paja canotuda & SD & SD & 10 & $\mathrm{~Pa}$ & eq, va \\
\hline \multicolumn{7}{|l|}{ POLYGONACEAE } \\
\hline Polygonum acuminatum Kunth, NDJ 2237 & duraznillo & $\mathrm{N}$ & Si & 15 & $\mathrm{~Pa}$ & va \\
\hline Ruprechtia apetala Wedd., GJM 865, 869 & chuluca, chicharra & $\mathrm{N}$ & $\mathrm{Si}$ & 15 & Ho & ca, va \\
\hline $\begin{array}{l}\text { PORTULACACEAE } \\
\text { Talinum paniculatum (Jacq.) } \\
\text { Gaertn., GJM 1178, NDJ } 2318\end{array}$ & carne gorda, verdolaga & A & $\mathrm{Si}$ & 15 & $\mathrm{~Pa}$ & po \\
\hline $\begin{array}{l}\text { PTERIDACEAE } \\
\text { Doryopteris triphylla (Lam.) } \\
\text { Christ, GJM 1277, } 1376\end{array}$ & helecho negrillo, negrillo & $\mathrm{N}$ & Si & 10 & $\mathrm{~Pa}$ & ov \\
\hline $\begin{array}{l}\text { RANUNCULACEAE } \\
\text { Anemone decapetala Ard. var. } \\
\text { decapetala, GJM 1312; NDJ } 1989\end{array}$ & cebolla del zorro & $\mathrm{N}$ & $\mathrm{Si}$ & 20 & PE & ca, po \\
\hline \multicolumn{7}{|l|}{ RHAMNACEAE } \\
\hline Colletia spinosissima J.F. Gmel., NDJ 2007 & tola, tola tola & $\mathrm{N}$ & $\mathrm{Cu}, \mathrm{Si}$ & 30 & $\mathrm{~Pa}$ & $\begin{array}{l}\text { ca, eq, } \\
\text { ov, va }\end{array}$ \\
\hline $\begin{array}{l}\text { Condalia buxifolia Reissek, } \\
\text { GJM 881, 940, } 1093\end{array}$ & piquillín & $\mathrm{N}$ & $\mathrm{Cu}, \mathrm{Si}$ & 15 & $\mathrm{~Pa}$ & ca, va \\
\hline $\begin{array}{l}\text { Condalia microphylla Cav., GJM } \\
945,1090 ; \text { NDJ } 2324\end{array}$ & piquillín & $\mathrm{N}$ & $\mathrm{Cu}, \mathrm{Si}$ & 15 & $\mathrm{~Pa}$ & ca, va \\
\hline Condalia montana A. Cast., GJM 1182, 1290 & piquillín & $\mathrm{N}$ & $\mathrm{Cu}, \mathrm{Si}$ & 15 & $\mathrm{~Pa}$ & ca, va \\
\hline $\begin{array}{l}\text { Sarcomphalus mistol (Griseb.) } \\
\text { Hauenschild, GJM } 944\end{array}$ & mistol & $\mathrm{N}$ & $\mathrm{Si}$ & 35 & $\mathrm{Fr}$, Ho & $\begin{array}{l}\text { ca, eq, ov, } \\
\text { po, va }\end{array}$ \\
\hline \multicolumn{7}{|l|}{ ROSACEAE } \\
\hline $\begin{array}{l}\text { Margyricarpus pinnatus (Lam.) } \\
\text { Kuntze, GJM 866, } 1261\end{array}$ & $\begin{array}{l}\text { manzanita, perlita, } \\
\text { huevito de la perdiz }\end{array}$ & $\mathrm{N}$ & $\mathrm{Pe}$ & 15 & $\mathrm{~Pa}$ & ca, ov, va \\
\hline Prunus persica (L.) Batsch, NDJ 2375 & durazno & $E$ & $\mathrm{Pe}$ & 10 & $\mathrm{Fr}$ & po \\
\hline \multicolumn{7}{|l|}{ RUTACEAE } \\
\hline Citrus reticulata Blanco, SC & mandarina, mandarino & $E$ & $\mathrm{Pe}$ & 10 & $\mathrm{Fr}$ & ca, ov \\
\hline \multicolumn{7}{|l|}{ SALICACEAE } \\
\hline Salix alba L. SC & mimbre & $E$ & $\mathrm{Pe}$ & 15 & $\mathrm{Fr}, \mathrm{Ho}$ & ca, eq, va \\
\hline Salix humboldtiana Willd., GJM 929 & sauce, sauce llorón & $\mathrm{N}$ & $\mathrm{Pe}$ & 30 & $\mathrm{Fr}, \mathrm{Ho}$ & ca, eq, va \\
\hline $\begin{array}{l}\text { SAPINDACEAE } \\
\text { Allophylus edulis (A. St.-Hil., } \\
\text { A. Juss. \& Cambess.) Hieron. } \\
\text { ex Niederl., GJM 899, } 956\end{array}$ & chal chal & $\mathrm{N}$ & Si & 30 & Ho & $\begin{array}{l}\text { ca, ov, } \\
\text { po, va }\end{array}$ \\
\hline $\begin{array}{l}\text { SCHIZAEACEAE } \\
\text { Anemia tomentosa (Savigny) Sw. } \\
\text { var. anthriscifolia (Schrad.) Mickel, } \\
\text { GJM } 877,1023,1201,1374\end{array}$ & doradilla & $\mathrm{N}$ & Si & 10 & PE & ca \\
\hline
\end{tabular}


Continuación Tabla 1

\begin{tabular}{|c|c|c|c|c|c|c|}
\hline FAMILIA, Especie, Vaucher & Nombre común & Origen & Hábitat & Fr\%* & Parte & Forraje \\
\hline $\begin{array}{l}\text { SCROPHULARIACEAE } \\
\text { Buddleja mendozensis Gillies ex } \\
\text { Benth. GJM 1029, 1273; NDJ } 2247\end{array}$ & salvia blanca & $\mathrm{N}$ & $\mathrm{Si}$ & 20 & $\mathrm{~Pa}$ & ca, eq, ov \\
\hline \multicolumn{7}{|l|}{ SIMAROUBACEAE } \\
\hline Castela coccinea Griseb., GJM 1167 & mistolillo & $\mathrm{N}$ & Si & 10 & $\mathrm{Fr}$ & ca \\
\hline \multicolumn{7}{|l|}{ SOLANACEAE } \\
\hline $\begin{array}{l}\text { Lycium cestroides Schltdl., GJM } \\
\text { 915, 1059; NDJ } 2267\end{array}$ & picoyuyo, piscoyuyo & $\mathrm{N}$ & Si & 25 & Ho & ca \\
\hline Nicotiana longiflora Cav., GJM 1045 & $\begin{array}{l}\text { flor de sapo, } \\
\text { yerba de sapo }\end{array}$ & $\mathrm{N}$ & Si & 10 & $\mathrm{~Pa}$ & ca \\
\hline $\begin{array}{l}\text { Petunia axillaris (Lam.) Britton, } \\
\text { Stern \& Poggenb. ssp. subandina } \\
\text { Ando, GJM 858, NDJ } 2290\end{array}$ & $\begin{array}{l}\text { bocinita, pepinia } \\
\text { del campo }\end{array}$ & $\mathrm{N}$ & $\mathrm{Si}$ & 10 & $\mathrm{~Pa}$ & ca, ov \\
\hline $\begin{array}{l}\text { Solanum argentinum Bitter \& } \\
\text { Lillo, GJM 951, } 1032\end{array}$ & malfato & $\mathrm{N}$ & $\mathrm{Si}$ & 20 & $\mathrm{~Pa}$ & eq \\
\hline Solanum chacoense Bitter, NDJ 2266 & papilla & $\mathrm{N}$ & $\mathrm{Si}$ & 10 & $\mathrm{Ra}$ & $\mathrm{eq}^{*}$ \\
\hline $\begin{array}{l}\text { Vassobia breviflora (Sendtn.) } \\
\text { Hunz., GJM 926, } 1060\end{array}$ & $\begin{array}{l}\text { piscoyuyo, } \\
\text { piscoyuyo blanco }\end{array}$ & $\mathrm{N}$ & $\mathrm{Si}$ & 15 & $\mathrm{~Pa}$ & av, ca \\
\hline \multicolumn{7}{|l|}{ URTICACEAE } \\
\hline Urtica circularis (Hicken) Sorarú, GJM 1314 & rupachico, ortiga & $\mathrm{N}$ & $\mathrm{Pe}$ & 15 & $\mathrm{~Pa}$ & av, ca, va \\
\hline \multicolumn{7}{|l|}{ VERBENACEAE } \\
\hline $\begin{array}{l}\text { Aloysia gratissima (Gillies \& Hook.) } \\
\text { Tronc., GJM 878, } 1175\end{array}$ & $\begin{array}{l}\text { palo amarillo, poleo } \\
\text { del campo }\end{array}$ & $\mathrm{N}$ & $\mathrm{Si}$ & 20 & $\mathrm{~Pa}$ & ca, ov, va \\
\hline Glandularia peruviana (L.) Small, GJM 857A & sangre de Cristo & $\mathrm{N}$ & $\mathrm{Cu}, \mathrm{Si}$ & 15 & PE & ca \\
\hline $\begin{array}{l}\text { Lippia juneliana (Moldenke) } \\
\text { Tronc., GJM 1085, NDJ } 2260\end{array}$ & salvia de campo, salvia & $\mathrm{N}$ & $\mathrm{Cu}, \mathrm{Si}$ & 40 & $\mathrm{~Pa}$ & ca, ov, va \\
\hline \multicolumn{7}{|l|}{ ZYGOPHYLLACEAE } \\
\hline $\begin{array}{l}\text { Porlieria microphylla (Baill.) Descole, } \\
\text { O'Donell \& Lourteig, GJM } 934\end{array}$ & $\begin{array}{l}\text { caspicuchara, frutilla } \\
\text { negra, monte crespo }\end{array}$ & $\mathrm{N}$ & $\mathrm{Si}$ & 20 & Ho & ca, ov \\
\hline \multicolumn{7}{|l|}{ INDET. } \\
\hline Indet. 1, NDJ 2313 & enredadera del sol & SD & SD & 10 & Ho & ca, ov, va \\
\hline
\end{tabular}

Referencias: Organizada en orden alfabético por familia botánica y nombre científico. Nombres comunes, sin nombre común (SN). Origen: exótica $(E)$, nativa $(N)$, sin información disponible (SD). Hábitat de la especie: cumbre $(\mathrm{Cu})$, sierra $(\mathrm{Se})$, peridoméstico $(\mathrm{Pe})$, venta comercial $(\mathrm{VC})$. Frecuencia de mención (Fr). Parte consumida: planta entera (PE), parte aérea (Pa), raíz (Ra), tallo (Ta), hoja (Ho), flor (FI), fruto (Fr), semilla (Se). Forraje: avícola (av), caprino (ca), equino (eq: equino solo burro eq*), ovino (ov), porcino (po), vacuno (va).

y ovino) sobresale el tamaño más pequeño de estos animales, que constantemente están pellizcando todo tipo de vegetación, que son capaces de comer plantas leñosas (principalmente las cabras) y que presentan bajos requerimientos de agua disponible, razones que le permiten a este tipo de ganado tolerar ambientes degradados, con suelos pobres o muy áridos.

En la relación entre las especies forrajeras y el tipo de ganado que las consume, se debe tener en cuenta factores como el extractivismo y la tala de bosques en el Chaco Seco. Como lo advierten Silvetti \& Cáceres (2006), en la actualidad se han generado procesos de sucesión secundaria en la vegetación, dando mayor dominio a especies espinosas. Éstas, presentan una calidad forrajera menor que las gramíneas, pero además dificultan o limitan el acceso del ganado a las pasturas, -incluso llegando a lacerar o herir los animales- siendo, el ganado vacuno y ovino los más perjudicados, pero no tanto así el caprino (Martínez \& JiménezEscobar, 2017). En general, es escasa la literatura científica disponible sobre la alimentación de las cabras con respecto a otros animales domésticos, razón que acrecienta la importancia de documentar las especies forrajeras y establecer el tipo de ganado que las consume.

En relación a la información que se obtuvo por 


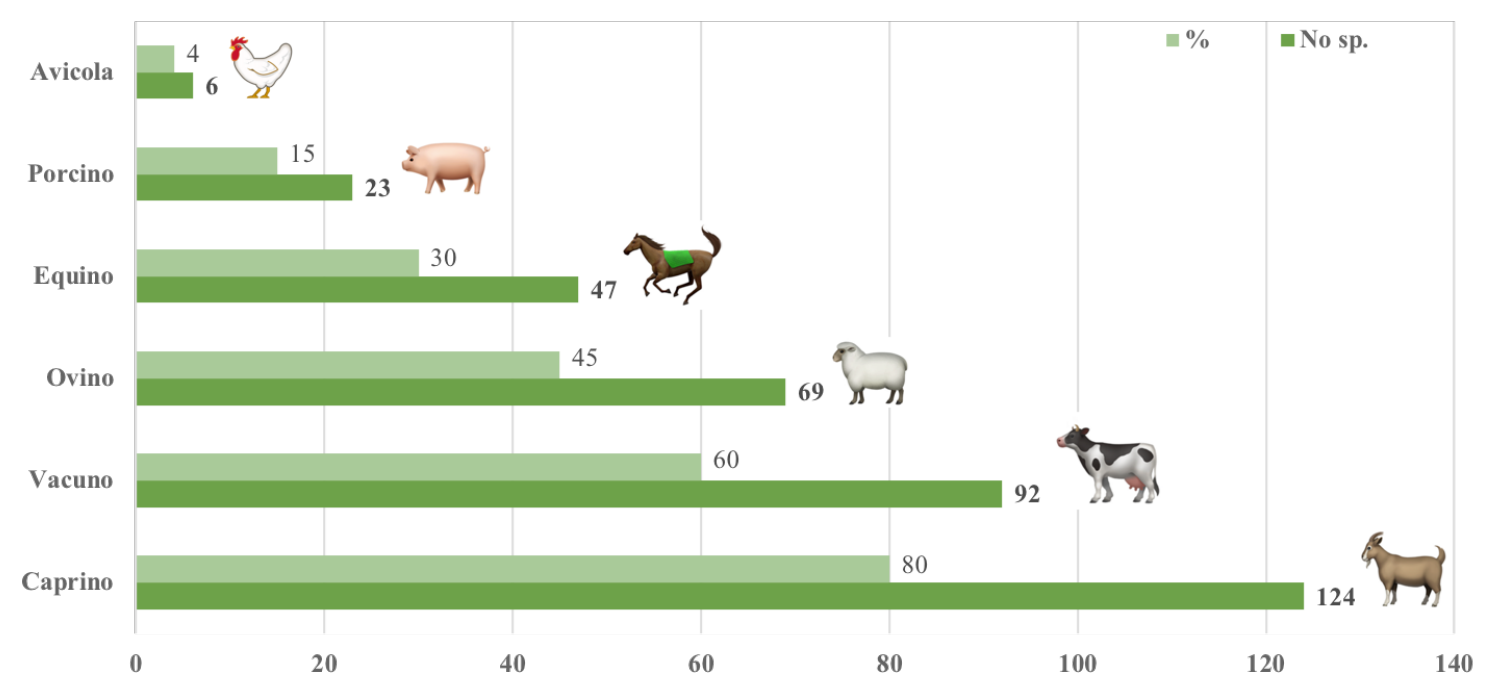

Fig. 2. Riqueza de especies vegetales consumidas por animales de cría en la Sierra de Ancasti, Catamarca.

los pobladores sobre las partes de las plantas que son forrajeadas, se encontró que la parte aérea de 88 especies es consumida por animales domésticos (Fig. 3). Este resultado se relaciona principalmente con pastos y otras herbáceas que son ramoneadas, pero donde específicamente no se consume la raíz (a diferencia de la categoría planta entera). Asimismo se destaca el consumo de las hojas de 30 especies y los frutos de otras 26 plantas, corroborando la amplia diversidad de los forrajes (en términos de riqueza de especies y partes consumidas).

\section{Frecuencia de mención}

En la lista comentada de las especies forrajeras (Tabla 1), se pueden consultar todos los valores obtenidos de la frecuencia relativa de Mención (Fr). Por otra parte, en la Tabla 2 se observan en detalle las especies con las mayores frecuencias de mención como forrajeras en Ancasti. El "alfa" (Medicago sativa) que se considera una especie adventicia -que además en la región es comprada y no es cultivada- fue la de mayor mención entre los pobladores. Seguida de "churqui" (Vachellia caven) una planta nativa ramoneada por los animales y el "maíz" (Zea mays) una gramínea comprada y cultivada en la región.

A fin de complementar la información de las especies con mayor frecuencia de mención, se incluyó la información observada en el campo sobre las principales formas de acceso y de disponibilidad de las mismas. De esta forma, de las especies más mencionadas por los criollos ( $\mathrm{Fr} \geq 35 \%$ ), sólo el "maíz" es cultivado en la zona. Pero, al igual que el "alfa", el fruto de esta gramínea puede ser adquirida en algunos de los almacenes o comercios de las áreas urbanas. Las restantes 15 especies son ramoneadas por los diferentes tipos de ganado. Si bien, el término ramonear se define como el corte de ramas, en este texto se utiliza para definir cuando el propio animal va en búsqueda del alimento y hace un consumo directo de cualquiera de las partes de la planta. Por último, entre las especies de mayor mención, siete son buscadas, recolectadas y llevadas por los criollos hasta los corrales y chiqueros para alimentar sus animales (Tabla 2). Estas especies corresponden a plantas nativas de la región.

Haber estimado la frecuencia relativa permitió esclarecer algunos detalles que se vinculan con la importancia cultural de las especies en una comunidad. Sin embargo, se debe aclarar que al indagar en las entrevistas sobre las especies forrajeras, las respuestas por parte de los pobladores fueron enfocadas a aquellas que se consideran como alimentos que mantienen al animal, principalmente en épocas de escasez. Es así como ninguna de las principales pasturas aparece entre las especies más mencionadas, mientras que las consideradas como de emergencia: Cereus forbesii, Ephedra tweediana, Geoffroea decorticans o Puya spathacea cobran mayor grado de visibilización y por ende de mención. 


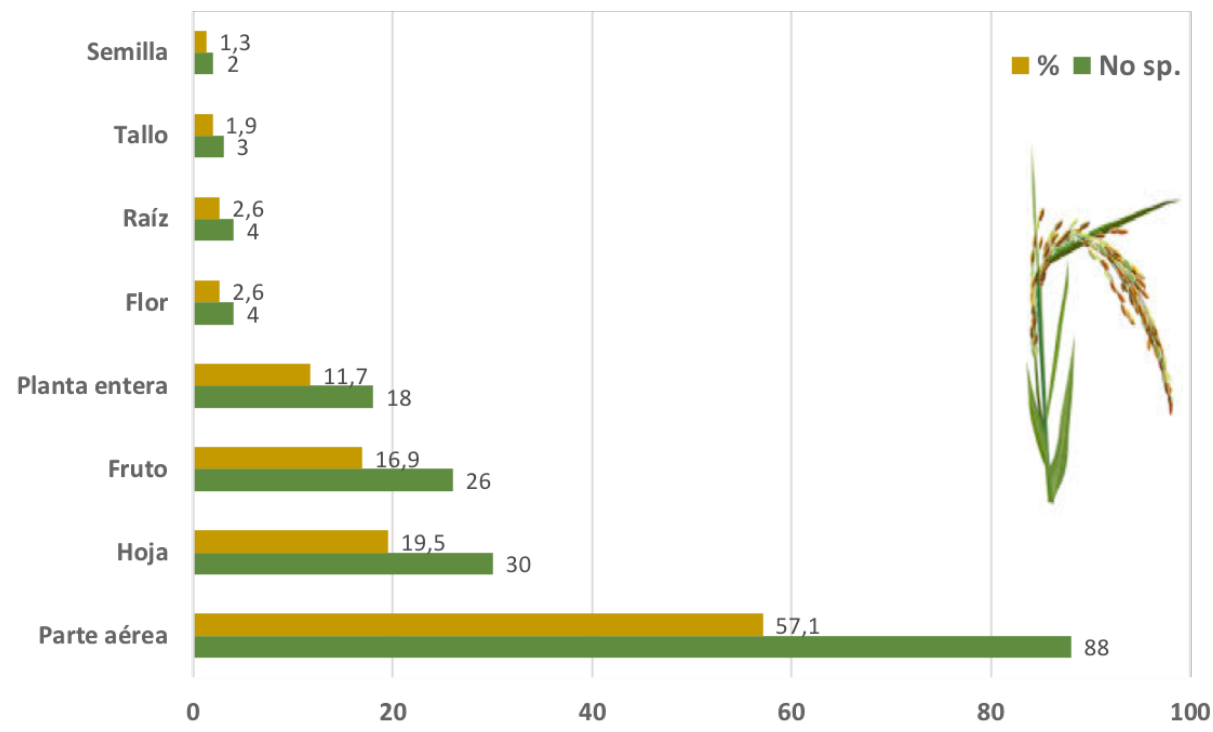

Fig. 3. Partes vegetales consumidas por los animales de cría en la Sierra de Ancasti, Catamarca.

Tabla 2. Especies y manejo de los principales forrajes según la frecuencia de mención (Fr.) entre los pobladores de la Sierra de Ancasti, Catamarca.

\begin{tabular}{|c|c|c|c|}
\hline $\begin{array}{l}\text { Nombre } \\
\text { común }\end{array}$ & Especie & Fr. (\%) & Manejo \\
\hline alfa & Medicago sativa & $15(75)$ & Co \\
\hline churqui & Vachellia caven & $13(65)$ & $\mathrm{Ra}$ \\
\hline maíz & Zea mays & $12(60)$ & $\mathrm{Co}, \mathrm{Cu}$ \\
\hline ucle & Cereus forbesii & $11(55)$ & $\mathrm{Ra}, \mathrm{Re}$ \\
\hline tusca & Vachellia aroma & $10(50)$ & $\mathrm{Ra}$ \\
\hline gramilla & Paspalum notatum & $9(45)$ & $\mathrm{Ra}$ \\
\hline huil & $\begin{array}{l}\text { Myrcianthes } \\
\text { cisplatensis }\end{array}$ & $9(45)$ & $\mathrm{Ra}, \mathrm{Re}$ \\
\hline shinqui & Mimosa farinosa & $9(45)$ & $\mathrm{Ra}$ \\
\hline tramontana & Ephedra tweediana & $9(45)$ & $\mathrm{Ra}, \mathrm{Re}$ \\
\hline $\begin{array}{l}\text { viscote } \\
\text { negro }\end{array}$ & Parasenegalia visco & $9(45)$ & $\mathrm{Ra}$ \\
\hline chañar & Geoffrea decortcans & $8(40)$ & $\mathrm{Ra}, \mathrm{Re}$ \\
\hline $\begin{array}{l}\text { salvia de } \\
\text { campo }\end{array}$ & Lippia juneliana & $8(40)$ & $\mathrm{Ra}$ \\
\hline azahar & Tillandsia spp. & $7(35)$ & $\mathrm{Ra}, \mathrm{Re}$ \\
\hline chaguar & Puya spathacea & $7(35)$ & $\mathrm{Ra}$ \\
\hline liga & $\begin{array}{c}\text { Ligaria cuneifolia, } \\
\text { Struthanthus } \\
\text { uraguensis }\end{array}$ & $7(35)$ & $\mathrm{Ra}, \mathrm{Re}$ \\
\hline mistol & Sarcomphalus mistol & $7(35)$ & $\mathrm{Ra}, \mathrm{Re}$ \\
\hline paja dura & Jarava ichu & $7(35)$ & $\mathrm{Ra}$ \\
\hline
\end{tabular}

Referencia: Frecuencia de mención (Fr., en 20 U.D.). Tipo de Manejo: comprado (Co), cultivado $(\mathrm{Cu})$, ramoneado $(\mathrm{Ra})$, recolectado $(\mathrm{Re})$.

\section{Conclusiones}

Durante casi tres siglos de asentamiento de las comunidades criollas en la Sierra de Ancasti, los pobladores han hecho parte activa de la construcción histórica de la región, y esta se asocia a la cría de animales. Diferentes aspectos geográficos y ecológicos se han venido articulando con lo social y de esta forma se ha establecido una cultura en torno al ganado. Cultura que atraviesa procesos de cambio, selección, transmisión, entre otras y que se puede evidenciar desde las formas en que los campesinos conocen, utilizan y aprovechan los recursos naturales.

La riqueza de especies consumidas por los distintos tipos de ganado, el dominio de las especies nativas en la alimentación de los animales y la variedad de tipos de hábito y partes de uso, son una muestra de la amplia diversidad biológica de plantas de la región. Esta información, de valor etnobiológico, es un primer paso al entendimiento de los sistemas pastoriles en una región cuyos ámbitos de subsistencia y estilos de vida locales mantiene estrechos vínculos con el ganado.

En la actualidad, los pobladores compran forrajes como maíz y alfa (principalmente en época invernal). Sin embargo, es claro que la diversidad de plantas contribuye al mantenimiento del ganado y permite a los campesinos ofrecer una dieta variada a los animales. Los resultados derivados 
de este estudio, pueden ser integrados al diseño de planes de manejo y a la implementación de sistemas silvopastoriles. Estrategias que garanticen a los pobladores, disponibilidad de forrajes, tanto en épocas de escasez de recursos vegetales como en periodos de bajos ingresos económicos.

Desde la biología, así como desde la antropología, resulta escasa la literatura científica que aborde temáticas donde se vinculen los procesos culturales, los animales de cría y las plantas forrajeras, siendo aún menor aquella relacionada al ganado caprino. Aunque este animal en muchos contextos es responsabilizado de la erosión de los suelos y la escasez de recursos vegetales, con frecuencia son las cabras para muchas de las sociedades campesinas, una fuente importante de ingresos económicos, así como parte fundamental del abastecimiento de carne y leche.

La inclusión de los procesos productivos y locales en la toma de decisiones es una forma en que la conservación puede ser promovida. La documentación que se obtenga sobre las especies forrajeras en términos de acceso, calidad, disponibilidad, diversidad, manejo, percepción y uso, representa un paso importante hacia la preservación de los conocimientos locales asociados al ámbito ganadero. Además, es clave a la hora de proponer y planear iniciativas de gestión sostenible y conservación de la biodiversidad, pensada hacia un mejoramiento de las prácticas de pastoreo y ganadería.

\section{Contribución DE LOS AUtORES}

NDJ estructuró la base de datos, preparó las tablas y las figuras, analizó y discutió los resultados y redactó el manuscrito. GJM realizó la revisión general del manuscrito. Ambos autores realizaron el trabajo de campo y las tareas de herbario.

\section{Agradecimientos}

Agradecemos a H. Sarmiento-Lepesqueur ${ }^{\dagger}$ por la lectura crítica de la primera versión del documento. A F. Pazzarelli por sus comentarios en el desarrollo del proyecto. A L. Ariza, G. Barboza, G.Bernardello, E. Cantero, R. Morero y C. Peichoto quienes colaboraron con la determinación de material vegetal. A N.Hilgert y dos evaluadores anónimos por sus acertados comentarios $\mathrm{y}$ sugerencias al manuscrito. Al IDACOR, Museo de Antropología, FFyH, UNC y al Herbario del Museo Botánico (CORD). Los conocimientos locales referidos en este documento pertenecen a la comunidad campesina de la Sierra de Ancasti, a quienes les agradecemos toda su colaboración. A los financiadores de esta investigación: Consejo Nacional de Investigaciones Científicas y Tecnológicas (CONICET) y al proyecto ANPCyT/ FONCyT (Pict 2012-1001) "Etnoecología y percepción ambiental en actores sociales para la conservación de áreas prioritarias del Chaco Serrano" dirigido por G.J. Martínez.

\section{Bibliografía}

ALBUQUERQUE, U.P., R.F.P. LUCENA \& N.L. ALENCAR. 2014. Methods and techniques used to collect ethnobiological data. In: ALBUQUERQUE, U.P., R.F.P. LUCENA \& L.V.F. CUNHA (eds.), Methods and techniques in ethnobiology and ethnoecology, pp. 15-37. Springer, New York, USA https://doi.org/10.1007/978-1-4614-8636-7_2

BAZÁN, A.R. 2006. Los pueblos de Ancasti. Editorial Sarquís, Catamarca, Argentina.

BRAUN WILKE, R. H. 1995. Plantas de interés ganadero de Jujuy y Salta Noroeste Argentino. Segunda edición. Universidad Nacional de Jujuy, Jujuy, Argentina.

BUGALLO, L. 2014. Flores para el ganado una concepción puneña del multiplico (Puna de Jujuy, Argentina). En: RIVERA, J.J. (ed.). Comprender los rituales ganaderos en los Andes y más allá, pp. 311-363. Bonner Amerikanistische Studien-BAS 51, Bonn, Alemania. https://doi.org/10.5565/rev/periferia.472

BURKART, A. 1943. Acantáceas indígenas como forrajeras de emergencia. Darwiniana 6: 192-202.

CÁCERES, D. 2006. El campesinado contemporáneo. En: CÁCERES, D., F. SILVETTI, G. FERRER \& G. SOTO (eds.). Y... vivimos de las cabras: transformaciones sociales y tecnológicas de la capricultura, pp. 23-46. Editorial La Colmena, Buenos Aires, Argentina.

CAVANNA, J., G. CASTRO, U. KARLIN \& M. KARLIN. 2010. Ciclo ganadero y especies forrajera en Salinas Grandes, Catamarca, Argentina. Zonas Áridas 14: 170-180. http://dx.doi.org/10.21704/za.v14i1.158 


\section{N. D. Jiménez-Escobar y G. J. Martínez - Plantas forrajeras de la Sierra de Ancasti, Catamarca}

DE RANCOURT, M., N. FOIS, M.P. LAVÍN, E. TCHAKÉRIAN \& F. VALLERAND. 2006. Mediterranean sheep and goat's production: An uncertain future. Small Rumin Res. 62: 167-179. http://dx.doi.org/10.1016/j.smallrumres. 2005.08.012

EVANS-PRITCHARD, E.E. 1940. The Nuer. Clarendon Press, Oxford.

FAO. 2011. Organización de las Naciones Unidas para la Agricultura y la Alimentación. State of the World's Forests. Rome, Italy. [En línea]. Disponible en: http://www.fao.org. [Acceso: 18 agosto 2018].

GALATY, J. \& D. JOHNSON. 1990. The word of pastoralism. Herding systems in comparative perspective. The Guilford Press, N.Y., London \& Belhaven Press, London.

https://doi.org/10.1017/S0032247400020465

GALEANO, L.M., M.J. GÓMEZ \& J.E. GÓMEZ. 2013. Caracterización de los sistemas de pastoreo de pequeños rumiantes en el sur del Tolima. Rev Colombiana Cienc Anim. 6: 74-84.

GIORGIS, M.A., A.M. CINGOLANI, F. CHIARINI, J. CHIAPELLA, G. BARBOZA, L. A. ESPINAR, R. MORENO, D.E. GURVICH, P.A. TECCO, R. SUBILS \& M. CABIDO. 2011. Composición florística del Bosque Chaqueño Serrano de la provincia de Córdoba, Argentina. Kurtziana 36: 9-43.

GUBER, R. 2011. La etnografía. Método, campo $y$ reflexividad. Siglo veintiuno editores, Buenos Aires, Argentina. http://dx.doi.org/10.5209/rev RASO. 2012\%20.v21.40380

GUBER, R. 2004. El salvaje metropolitano, Reconstrucción del conocimiento social en el trabajo de campo. Editorial Paidos, Buenos Aires, Argentina.

INDEC. 2010. Instituto Nacional de Estadística y Censos. República de Argentina. [En línea]. Disponible en: http://www.indec.gov.ar [Acceso: 15 abril 2015].

ISE. 2006. International Society of Ethnobiology. Code of Ethics (with 2008 additions). [En línea]. Disponible en: http://ethnobiology.net/code-of-ethics/ [Acceso: 13 septiembre 2014].

JIMÉNEZ-ESCOBAR, N.D. 2019. Etnobotánica asociada al ámbito ganadero: conocimiento, uso y conservación de los recursos vegetales en las Sierras de Ancasti (Catamarca). Tesis Doctoral, Facultad de Ciencias Exactas, Fisicas y Naturales, Universidad Nacional de Córdoba, Córdoba. Argentina. Pp 244.

JIMÉNEZ-ESCOBAR, N.D. \& G.J. MARTÍNEZ. 2019. Firewood knowledge, use and selection by rural populations in the Dry Chaco of Sierra de Ancasti, Catamarca, Argentina. Ethnobiol. Conserv. 8: 1-19 http://dx.doi.org/10.15451/ec2019-01-8.03-1-19

JIMÉNEZ-ESCOBAR, N.D. 2015. Entre "azahares" y "chaguares": las bromelias forrajeras en las Sierras de Ancasti, Catamarca, Argentina. Gaia Scientia 9: 1-6.

KARREMANS, J. 1994. Sociología para el desarrollo, métodos de investigación y técnicas de la entrevista. Serie técnica, informe técnico $\mathrm{N}^{\circ} 228$. Centro Agronómico Tropical de Investigación y Enseñanza (CATIE), Turrialba, Costa Rica.

LEDESMA, R., F. SARACCO, R.D. CORIA, F. EPSTEIN, A. GOMEZ, C. KUNST, M. ÁVILA \& J.F. PENSIERO. 2017. Guía de forrajeras herbáceas y leñosas del Chaco Seco: identificación y características para su manejo. Buenas prácticas para una ganadería sustentable. Kit de extensión para el Gran Chaco. Fundación Vida Silvestre Argentina. Buenos Aires, Argentina.

MARINO, G. 2013. Pastizales y sabanas del Cono Sur de Sudamérica. Iniciativas para su manejo $y$ Conservación. Temas de Naturaleza y Conservación, Monografia de Aves Argentinas № 9. Aves Argentinas/ AOP, Fundación Vida Silvestre Argentina e Instituto Nacional de Tecnología Agropecuaria. Buenos Aires, Argentina.

MARINO, G. 2008. Una ganadería diferente en los pastos naturales. [En línea]. Disponible en: http://www. produccion-animal.com.ar/produccion_y_manejo_ pasturas/pasturas\%20naturales/126-diferente.pdf [Acceso: 23 enero 2019].

MARTÍNEZ, G. \& N. D. JIMÉNEZ-ESCOBAR. 2017. Plantas de interés veterinario en la cultura campesina de la Sierra de Ancasti (Catamarca, Argentina). BLACPMA 16: $329-346$.

MORELLO, J., I. FELDMAN \& I. GÓMEZ. 1973. La integración de la actividad agro-silvo-pastoril en el centro oeste de Formosa (Chaco Argentino). IDIA 305: 17-37.

MORELlO, J. \& C. SARAVIA. 1959a. El bosque chaqueño 1. Paisaje primitivo, paisaje natural y paisaje cultural en el Oriente de Salta. Revista Agron. Noroeste Argent. 3: 5-81.

MORELlO, J. \& C. SARAVIA. 1959b. El bosque chaqueño 2. La ganadería y el bosque en el Oriente de Salta. Revista Agron. Noroeste Argent. 3: 209258.

MORI, S. A., A. BERKOV, C.A. GRACIE \& E.F. HECKLAU. 2011. Tropical Plant Collecting. From the Field to the Internet. TECC Editora, Florianópolis, Brasil. 
MORLÁNS, M.C. 1995. Regiones Naturales de Catamarca, Provincias Geológicas y Provincias Fitogeográficas. Revista de Ciencia y Técnica 2:142.

MUIÑO, W.A. 2010. Etnobotanical study of the rural population of the west of the pampa plain (Argentina). Ethnobot. Res. App. 8: 219-231.

NUNES, A.T., R.F.P. LUCENA, M.V.F. DOS SANTOS \& U.P. ALBUQUERQUE. 2015. Local knowledge about fodder plants in the semi-arid region of Northeastern Brazil. J Ethnobiol. Ethnomed. 11: 1-12. https://doi.org/10.1186/1746-4269-11-12

QUIROGA, A. \& R.J. CORREA. 2011. Gramineas forrajeras presentes en el Chaco Árido de Catamarca. Revista de Divulgación Técnica Agrícola y Agroindustrial. Facultad de Ciencias Agrarias, Universidad Nacional de Catamarca, 16: $1-12$.

QUIROGA, A. \& G. REINOSO-FRANCHINO. 2010. Árboles nativos de la provincia de Catamarca. Atlas Catamarca. Gobierno de la Provincia de Catamarca, Facultad de Ciencias Agrarias. [En línea]. Disponible en: http://www.atlas.catamarca. gov.ar [Acceso: 01 septiembre 2017].

QUIROGA MENDIOLA, M. 2011. Sociedades y agroecosistemas pastoriles de alta montaña en la Puna. Departamento de Yavi, provincia de Jujuy, República de Argentina. Tesis Doctoral, Facultad de Ciencias Agropecuarias. Universidad Nacional de Córdoba, Córdoba, Argentina. Pp. 212.

PALMIERI, C.N., I.M. CARMA \& A. QUIROGA. 2008. La Ecorregiones de Catamarca. Atlas de Catamarca. Universidad Nacional de Catamarca, Facultad de Ciencias Agrarias. [En línea]. Disponible en: http:// www.atlas.catamarca.gov.ar [Acceso: 01 septiembre 2017].

PEREA, M.V. 2011. Relevamiento de Cactáceas en la provincia de Catamarca. Consejo Fed. de Inversiones, Buenos Aires, Argentina.

PEREA, M.V., G. PEDRAZA \& J. LUCEROS. 2007. Relevamiento de la flora arbórea autóctona de la provincia de Catamarca. Consejo Federal de Inversiones, Buenos Aires, Argentina.

RAGONESE, A.E. \& V.A. MILANO. 1984. Vegetales y sustancias tóxicas de la flora Argentina. Enciclopedia Argentina de Agricultura y Jardinería. 2o Ed. Tomo II, Fasc. 8-2. Ed. ACME. Buenos Aires, Argentina.

RAGONESE, A.E. 1967. Vegetación y ganadería en la República Argentina. INTA, Buenos Aires, Argentina.
RAMISCH, G., P. GHIONE, M. QUIROGAMENDIOLA, L. BILBAO \& F. CHAVEZ. 2009. Un acercamiento al papel de las políticas sociales en la persistencia de pequeños productores pobres, el caso de Ancasti, Catamarca. En: RAMILO, D. \& G. PRIVIDERA. La agricultura familiar en la Argentina. Diferentes abordajes para su estudio. INTA. Estudios socioeconómicos de los sistemas agroalimentarios y agroindustriales 20: pp. 115132. [En línea]. Disponible en: https://inta.gob.ar/ sites/default/files/script-tmp-inta-ipafnoa-2013 ancasti.pdf [Acceso: 31 julio 2017].

RIAT, P. 2012. Conocimiento campesino, el "monte santiagueño" como recurso forrajero. Trab. soc. 19: 477-491.

RIVERA, J.J. 2014. ¿Qué son los rituales ganaderos? El tratamiento de animales en los Andes contemporáneos. En: RIVERA, J.J. (ed.). Comprender los rituales ganaderos en los Andes y más allá. Bonner Amerikanistische StudienBAS 51, Bonn, Alemania.

ROIG, C. 2003. Alimentación del ganado caprino. Informe técnico: PROGANO-INTA. Colonia Benítez, Chaco, Argentina.

SÁNCHEZ, T., M. MILERAS, L. SIMÓN, L. LAMELA \& O. LÓPEZ. 2007. Las potencialidades de las asociaciones Gramíneas-Leguminosas como alimento de los rumiantes. REDVET Revista Electrónica de Veterinaria 8 (12): 1-10.

SCARPA, G.F. 2012. Las plantas en la vida de los criollos del oeste formoseño: medicina, ganadería, alimentación y viviendas tradicionales. Asociación Civil Rumbo Sur, Buenos Aires, Argentina.

SCARPA, G.F. 2007. Etnobotánica de los Criollos del oeste de Formosa: Conocimiento tradicional, valoración y manejo de las plantas forrajeras. Kurtziana 33: 154-174.

SCHWABE, C.W. \& I.M. KUOJOK. 1981. Practices and beliefs of the traditional Dinka healer in relation to provision of modern medical and veterinary services for the Southern Sudan. Hum. Organ. 40: 231-238.

SILVETTI, F. \& D. CÁCERES. 2006. Una perspectiva socio histórica de las estrategias campesinas del Norte de Córdoba. En: CÁCERES, D., F. SILVETTI, G. FERRER \& G. SOTO (eds.), $Y \ldots$ vivimos de las cabras: transformaciones sociales $y$ tecnológicas de la capricultura, pp. 47-74. Editorial La Colmena, Buenos Aires. 


\section{N. D. Jiménez-Escobar y G. J. Martínez - Plantas forrajeras de la Sierra de Ancasti, Catamarca}

UICN. 2008. The International Union for Conservation of Nature. Policies that work for Pastoral environments. The World Initiative for Sustainable Pastoralism. Nairobi, Kenia. [En línea]. Disponible en: http://cmsdata.iucn. org/downloads/goa_uicn_wisp_policies_and_pastoral_ environments_en.pdf)[Acceso: 02 mayo 2018].
ZULOAGA, F.O., O. MORRONE \& M.J. BELGRANO. 2008. Catálogo de Plantas Vasculares del Cono Sur. Monogr. Missouri Bot. Garden (USA). [En línea]. Disponible en: http://www2.darwin.edu. ar/proyectos/floraargentina/Generos [Acceso: 18 septiembre 2018]. 
